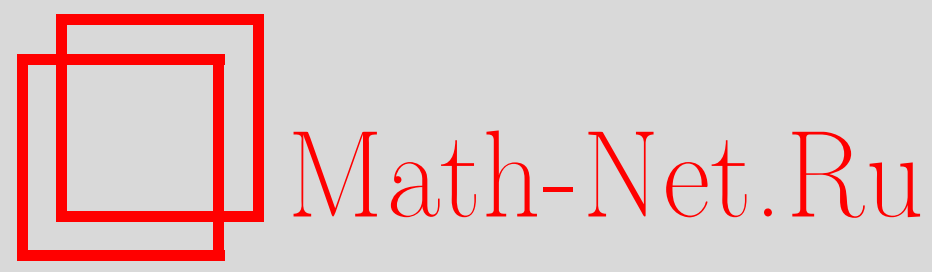

М. Е. Жуковский, Оценка количества максимальных расширений в случайном графе, Дискрет. матем., 2012, том 24, выпуск 1, 79-107

DOI: https://doi.org/10.4213/dm1174

Использование Общероссийского математического портала Math-Net.Ru подразумевает, что вы прочитали и согласны с пользовательским соглашением http://www.mathnet.ru/rus/agreement

Параметры загрузки:

IP: 3.89 .197 .203

26 апреля 2023 г., 09:34:12 


\title{
Оценка количества максимальных расширений в случайном графе
}

\author{
(c) 2012 г. М. Е. Жуковский
}

\begin{abstract}
В работе изучается классическая задача об асимптотическом поведении вероятностей свойств случайных графов в модели, впервые рассмотренной П. Эрдешем и А. Реньи. Исследуются различные расширения подграфов случайного графа. Число так называемых надежных расширений подграфов в случайном графе было оценено в 1990 г. Дж. Спенсером. В его работе был поставлен вопрос, для каждого ли набора вершин существуют расширения, которые в определенном смысле не расширяются дальше. Мы ввели понятия нейтральной и максимальной пары, оценили число максимальных пар и дали тем самым исчерпывающий ответ на поставленный Спенсером вопрос.

Задача о числе расширений находит свое применение в обосновании справедливости законов нуль-единица для случайных графов. Наш результат также используется для доказательства некоторых таких законов.

В исследуемой нами задаче о распределении малых подграфов в случайном графе до сих пор оставалась одна неразрешенная ситуация, которую мы и рассмотрели. Таким образом, наша теорема не только служит новым инструментом в доказательстве законов нуль-единица, но и закрывает брешь в изучении малых подграфов в случайном графе.
\end{abstract}

\section{1. Введение}

В работе изучаются различные расширения подграфов случайного графа. Число так называемых надежных расширений подграфов в случайном графе было оценено в 1990 г. Дж. Спенсером (см. $[1,2])$. В отдельных случаях возникает вопрос, для каждого ли набора вершин существуют расширения данного типа, которые в определенном смысле не расширяются дальше. Для рассмотрения этих случаев мы введем понятия нейтральной пары и максимального расширения, после чего оценим число максимальных расширений.

Задача о числе расширений находит свое применение в обосновании справедливости законов нуль-единица для случайных графов (см. [2-6]). Впервые такой подход был использован в работе Дж. Спенсера и С. Шела в 1988 г. [3]. В этой работе получены результаты для свойств, записанных на языке первого порядка (см. $[7,8])$. Кроме того, в 1997 г. М. МакАртур (см. [4]) рассмотрела свойства, заданные формулами с неограниченным числом дизъюнкций и конъюнкций и с ограниченной заранее фиксированным числом кванторной глубиной. Наши результаты применяются для доказательства законов нуль-единица для свойств, записанных на языке первого порядка, при этом глубина формул опять же ограничена заранее фиксированным числом. Для таких свойств нам удалось расширить результат Дж. Спенсера и С. Шела. 
В следующем параграфе мы приведем формулировку теоремы Спенсера, а также введем все необходимые для этого определения. Кроме того, мы дадим определения нейтральной и максимальной пары графов, которые понадобятся, чтобы сформулировать в следующем разделе полученные нами результаты о максимальных расширениях. Доказательство этих теорем мы приведем в разделе 3 . Помимо этого, в разделе 4 рассматриваются некоторые обобщения этих теорем.

\section{1. Основные определения. Теорема о числе надежных расширений}

Как обычно, граф $G=(V, E)$ состоит из множества вершин $V$ и совокупности ребер $E$ (см. [9, 10]). Мы используем также обозначения $V=V(G)$ и $E=E(G)$. Рассмотрим графы $H, G, \widetilde{H}, \widetilde{G}$ такие, что $V(H)=\left\{x_{1}, \ldots, x_{k}\right\}, V(G)=\left\{x_{1}, \ldots, x_{l}\right\}, V(\widetilde{G})=\left\{\tilde{x}_{1}, \ldots, \tilde{x}_{l}\right\}$, $V(\tilde{H})=\left\{\tilde{x}_{1}, \ldots, \tilde{x}_{k}\right\}$, причем $H \subset G, \widetilde{H} \subset \widetilde{G}$ (в силу этого, $k<l$ ).

Граф $\widetilde{G}$ называется $(G, H)$-расширением графа $\widetilde{H}$, если

$$
\left\{x_{i}, x_{j}\right\} \in E(G) \backslash E(H) \Longrightarrow\left\{\tilde{x}_{i}, \tilde{x}_{j}\right\} \in E(\tilde{G}) \backslash E(\tilde{H})
$$

(здесь и далее ребро, соединяющее вершины $x$ и $y$, записывается как $\{x, y\}$ ). Если выполняется соотношение

$$
\left\{x_{i}, x_{j}\right\} \in E(G) \backslash E(H) \Longleftrightarrow\left\{\tilde{x}_{i}, \tilde{x}_{j}\right\} \in E(\widetilde{G}) \backslash E(\tilde{H}),
$$

то $\widetilde{G}$ назовем точным расширением. Положим

$$
v(G, H)=|V(G) \backslash V(H)|, \quad e(G, H)=|E(G) \backslash E(H)| ;
$$

(здесь и далее $|X|$ есть мощность множества $X$ ). Пусть фиксировано число $\alpha>0$. Если для любого такого графа $S$, что $H \subset S \subseteq G$, выполнено неравенство $v(S, H)-\alpha e(S, H)>0$, то пара $(G, H)$ называется $\alpha$-надежной (см. $[1,2,5])$. Если же для любого такого графа $S$, что $H \subseteq S \subset G$, выполнено неравенство $v(G, S)-\alpha e(G, S)<0$, то пара $(G, H)$ называется $\alpha$-жесткой (см. $[2,5])$.

Напомним теперь определение случайного графа. Пусть $N$ является натуральным числом, $0 \leqslant p \leqslant 1$. Рассмотрим множество $\Omega_{N}=\{\mathscr{G}=(\mathscr{V}, \mathscr{E})\}$ всех неориентированных графов без петель и кратных ребер с множеством вершин $\mathscr{V}=\{1, \ldots, N\}$. Случайный граф - это вероятностное пространство

$$
G(N, p)=\left(\Omega_{N}, \mathscr{F}_{N}, \mathbf{P}_{N, p}\right),
$$

где

$$
\mathscr{F}_{N}=2^{\Omega_{N}}, \quad \mathbf{P}_{N, p}(\mathscr{G})=p^{|\mathscr{E}|}(1-p)^{\left(\begin{array}{c}
N \\
2
\end{array}\right)-|\mathscr{E}|} .
$$

Иными словами, любые две различные вершины графа в пространстве $G(N, p)$ соединены ребром с вероятностью $p$ независимо от всех остальных пар вершин (см. [5, 11-13]). В дальнейшем мы будем рассматривать модели, в которых вероятность $p$ зависит от числа вершин $N$. В данной работе нас будет интересовать асимптотическое поведение вероятностей выполнения свойств случайных графов (то есть для каждого свойства $\mathscr{A}-$ асимптотическое поведение величин $\mathbf{P}_{N, p}\left(\mathscr{Y}_{N}\right)$, где $\mathscr{S}_{N} \in \mathscr{F}_{N}-$ множество всех графов из $\Omega_{N}$, обладающих свойством $\left.\mathscr{A}\right)$.

Пусть $\mathscr{A}-$ некоторое свойство и $\mathscr{S}_{N}-$ множество всех графов из $\Omega_{N}$, обладающих этим свойством. Мы будем говорить, что свойство $\mathscr{A}$ выполнено с асимптотической 


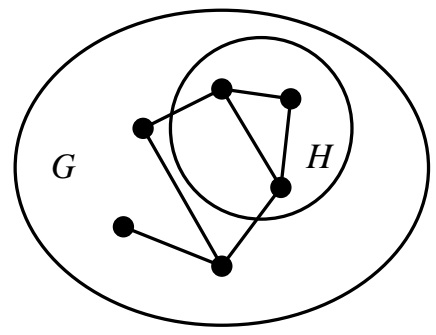

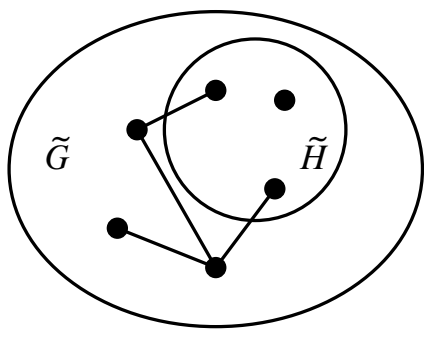

точное $(G, H)$-расширение

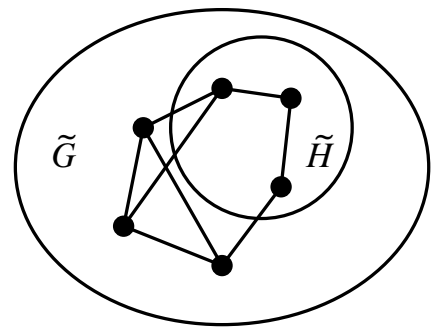

$(G, H)$-расширение

Рис. 1.

вероятностью 1, если вероятность его выполнения стремится к 1, то есть справедливо равенство

$$
\lim _{N \rightarrow \infty} \mathbf{P}_{N, p}\left(\mathscr{S}_{N}\right)=1 \text {. }
$$

Будем, кроме того, называть функцию $p_{0}(N)$ пороговой для свойства $L$, если

$$
\begin{array}{ll}
\lim _{N \rightarrow \infty} \mathbf{P}_{N, p}(G \vDash L)=1, & p=o\left(p_{0}\right), \\
\lim _{N \rightarrow \infty} \mathbf{P}_{N, p}(G \vDash L)=0, & p=\omega\left(p_{0}\right) .
\end{array}
$$

Здесь и далее для функций $f=f(N), g=g(N)$ мы будем говорить, что справедливо равенство $f=\omega(g)$ при $N \rightarrow \infty$ в том случае, когда для любого числа $C>0$ найдется такое $N_{0}$, что для всех $N>N_{0}$ выполнено неравенство $|f(N)|>C|g(N)|$.

Рассмотрим случайный граф $G(N, p(N))=\left(\Omega_{N}, \mathscr{F}_{N}, \mathbf{P}_{N, p}\right)$ и два графа $H$ и $G$ таких, что $H \subset G, V(H)=\left\{x_{1}, \ldots, x_{k}\right\}, V(G)=\left\{x_{1}, \ldots, x_{l}\right\}$. Пусть $\tilde{x}_{1}, \ldots, \tilde{x}_{k} \in \mathscr{V}$ и случайная величина $N_{(G, H)}\left(\tilde{x}_{1}, \ldots, \tilde{x}_{k}\right)$ на вероятностном пространстве $G(N, p(N))$ каждому графу $\mathscr{G}$ из $\Omega_{N}$ ставит в соответствие число $(G, H)$-расширений подграфа в $\mathscr{G}$, индуцированного на $\left\{\tilde{x}_{1}, \ldots, \tilde{x}_{k}\right\}$ (граф $X$ является подграфом графа $Y$, индуцированным на множество $S \subset V(Y)$, если $V(X)=S$ и для любых вершин $x, y \in S$ справедливо соотношение $\{x, y\} \in E(X) \Longleftrightarrow\{x, y\} \in E(Y))$. Иными словами, пусть $W \subset \mathscr{V} \backslash\left\{\tilde{x}_{1}, \ldots, \tilde{x}_{k}\right\}-$ множество, мощность $|W|$ которого равна $l-k$. Если можно так занумеровать элементы множества $W$ числами $k+1, k+2, \ldots, l$, что граф $\left.\mathscr{G}\right|_{\left\{\tilde{x}_{1}, \ldots, \tilde{x}_{l}\right\}}$ является $(G, H)$-расширением графа $\left.\mathscr{G}\right|_{\left\{\tilde{x}_{1}, \ldots, \tilde{x}_{k}\right\}}$, то положим $I_{W}(\mathscr{G})=1$. В противном случае $I_{W}(\mathscr{G})=0$. Случайная величина $N_{(G, H)}\left(\tilde{x}_{1}, \ldots, \tilde{x}_{k}\right)$ определяется равенством

$$
N_{(G, H)}\left(\tilde{x}_{1}, \ldots, \tilde{x}_{k}\right)=\sum_{\substack{W \subset \mathcal{V} \backslash\left\{\tilde{x}_{1}, \ldots, \tilde{x}_{k}\right\} \\|W|=l-k}} I_{W} .
$$


Теорема 1 ([1,2]). Пусть $p=N^{-\alpha}$ и пара $(G, H)$ является $\alpha$-надежной. Тогда для любого $\varepsilon>0$ справедливо равенство

$$
\begin{aligned}
\lim _{N \rightarrow \infty} \mathbf{P}_{N, p}\left(\left|N_{(G, H)}\left(\tilde{x}_{1}, \ldots, \tilde{x}_{k}\right)-\mathbf{E}_{N, p} N_{(G, H)}\left(\tilde{x}_{1}, \ldots, \tilde{x}_{k}\right)\right|\right. & \\
& \left.\leqslant \varepsilon \mathbf{E}_{N, p} N_{(G, H)}\left(\tilde{x}_{1}, \ldots, \tilde{x}_{k}\right)\right)=1
\end{aligned}
$$

для всех $\tilde{x}_{1}, \ldots, \tilde{x}_{k}$, где $\mathbf{E}_{N, p}$ - математическое ожидание по мере $\mathbf{P}_{N, p}$.

Замечание 1. Утверждение этой теоремы Спенсера фактически означает, что с асимптотической вероятностью 1 для любых вершин $\tilde{x}_{1}, \ldots, \tilde{x}_{k}$ справедливо соотношение

$$
N_{(G, H)}\left(\tilde{x}_{1}, \ldots, \tilde{x}_{k}\right) \sim \mathbf{E}_{N, p} N_{(G, H)}\left(\tilde{x}_{1}, \ldots, \tilde{x}_{k}\right)
$$

при $N \rightarrow \infty$. В дальнейшем в подобных случаях мы будем пользоваться именно такой формой записи.

Замечание 2. Справедливо равенство

$$
\mathbf{E}_{N, p} N_{(G, H)}\left(\tilde{x}_{1}, \ldots, \tilde{x}_{k}\right)=\left(\begin{array}{c}
N \\
v(G, H)
\end{array}\right) p^{e(G, H)} .
$$

Поэтому

$$
\mathbf{E}_{N, p} N_{(G, H)}\left(\tilde{x}_{1}, \ldots, \tilde{x}_{k}\right)=\Theta\left(N^{v(G, H)-\alpha e(G, H)}\right)
$$

и стремится к бесконечности при $N \rightarrow \infty$ (для функций $f=f(N), g=g(N)$, мы говорим, что $f=\Theta(g)$ при $N \rightarrow \infty$, если существуют такие положительные числа $c, C$ и $N_{0} \in \mathbf{N}$, что для всех $N>N_{0}$ справедливы неравенства $\left.c|f(N)| \leqslant|g(N)| \leqslant C|f(N)|\right)$. Действительно, в показателе степени стоит положительное число $v(G, H)-\alpha e(G, H)$ в случае $\alpha$-надежной пары $(G, H)$. Поэтому в этом случае с асимптотической вероятностью 1 для любых вершин $\tilde{x}_{1}, \ldots, \tilde{x}_{k} \in \mathscr{V}(G, H)$-расширение графа, индуцированного на множество $\left\{\tilde{x}_{1}, \ldots, \tilde{x}_{k}\right\}$, существует, и число таких расширений с ростом $N$ стремится к бесконечности.

Заметим, что если $\alpha$ - рациональное число, то равенство $v(G, H)-\alpha e(G, H)=0$ возможно. Рассмотрим, скажем, случайный граф с $p=N^{-\alpha}, \alpha=1 / 2$. Пусть $G-$ полный граф на трех вершинах, $H$ - подграф, индуцированный на некоторые его две вершины. Найдем вероятность того, что для конкретных двух вершин $\tilde{x}_{1}, \tilde{x}_{2}$ найдется третья вершина $\tilde{x}_{3}$, соединенная с каждой из них. Она, очевидно, равна

$$
1-\left(1-p^{2}\right)^{N-2} \sim 1-1 / e .
$$

Поэтому

$$
\mathbf{P}_{N, p}\left(N_{(G, H)}\left(\tilde{x}_{1}, \tilde{x}_{2}\right)=0\right) \sim 1 / e
$$

при $N \rightarrow \infty$. Кроме того, при достаточно больших $N$ справедливо неравенство

$$
\mathbf{E}_{N, p} N_{(G, H)}\left(\tilde{x}_{1}, \tilde{x}_{2}\right) \geqslant 1-2 / e .
$$

Таким образом, утверждение теоремы не выполнено. Мы показали, что с асимптотической вероятностью 1 для каждой вершины $\tilde{x}_{1}$ найдется вершина $\tilde{x}_{2}$, для которой не существует вершины $\tilde{x}_{3}$, соединенной с каждой из $\tilde{x}_{1}, \tilde{x}_{2}$. Иными словами, для расширения на 
вершинах $\tilde{x}_{1}, \tilde{x}_{2}$ графа на одной вершине $\tilde{x}_{1}$ не существует описанного расширения на вершинах $\tilde{x}_{1}, \tilde{x}_{2}, \tilde{x}_{3}$. При этом с асимптотической вероятностью 1 для каждой вершины $\tilde{x}_{1}$ число таких «вторых» вершин $\tilde{x}_{2}$ эквивалентно $\mathrm{Ne} e^{-1}$.

Образно говоря, мы рассмотрели расширения, которые в некотором смысле не расширяются дальше, то есть являются максимальными, и оценили их число в случайном графе. Как отмечалось выше, эти расширения используются для доказательств законов нуль-единица для случайных графов. В приведенном примере при $\alpha<1 / 2$ или $\alpha>1 / 2$ такие оценки напрямую следуют из результатов, полученных Дж. Спенсером (см. [1, 2, 5]). Опишем подробнее эти расширения.

\section{2. Нейтральные и максимальные пары}

Пусть $0<\alpha \leqslant 1-$ рациональное число. Рассмотрим графы $H, G$. Пусть $H \subset G$, любая вершина графа $H$ соединена с некоторой вершиной из $V(G) \backslash V(H)$, и для любого такого графа $S$, что $H \subset S \subset G$, справедливо неравенство $v(S, H)-\alpha e(S, H)>0$, но $v(G, H)-\alpha e(G, H)=0$. В этом случае пару $(G, H)$ будем называть $\alpha$-нейтральной. Заметим, что условие $0<\alpha \leqslant 1$ существенно. Оно необходимо для существования нейтральной пары. Действительно, пусть пара $(G, H)$ является $\alpha$-нейтральной. Пусть, кроме того, для некоторых вершин $y \in V(G) \backslash V(H), x \in V(H)$, справедливо соотношение $y \sim x$. Такие вершины найдутся в силу определения нейтрального расширения. Пусть $S-$ подграф графа $G$, индуцированный на множество вершин $\{y\} \cup V(H)$. Тогда $v(S, H)=e(S, H)=1$, но $\alpha \leqslant v(S, H) / e(S, H)$. Поэтому $\alpha \leqslant 1$.

Пусть $\widetilde{H} \subset \widetilde{G} \subset \Gamma$ и $T \subset K$, причем $|V(T)| \leqslant|V(\widetilde{G})|$. Пара $(\tilde{G}, \tilde{H})$ называется $(K, T)$ максимальной в $\Gamma$, если у любого подграфа $\widetilde{T}$ графа $\widetilde{G}$ такого, что $|V(\widetilde{T})|=|V(T)|$ и $\widetilde{T} \cap \tilde{H} \neq \widetilde{T}$, не существует такого точного $(K, T)$-расширения $\widetilde{K}$ в $\Gamma \backslash(\widetilde{G} \backslash \widetilde{T})$, что каждая вершина из $V(\widetilde{K}) \backslash V(\widetilde{T})$ не соединена ребром ни с одной вершиной из $V(\widetilde{G}) \backslash V(\widetilde{T})$. Заметим, что если $|V(T)|>|V(\widetilde{H})|$, то условие $\widetilde{T} \cap \widetilde{H} \neq \widetilde{T}$ не требуется.

Для наглядности рассмотрим пример максимальной пары в некотором графе Г. Пусть граф $\widetilde{H} \subset \Gamma$ состоит из одной вершины $\tilde{x}_{1}$, полный граф $\widetilde{G} \subset \Gamma$ на вершинах $\tilde{x}_{1}, \tilde{x}_{2}, \tilde{x}_{3}$ содержит в себе граф $\tilde{H}$. Пусть, кроме того, $K-$ граф на вершинах $y_{1}, y_{2}, y_{3}, T-$ его подграф на вершинах $y_{1}, y_{2}$, причем в графе $K$ вершина $y_{3}$ соединена с вершинами $y_{1}$, $y_{2}$. Тогда пара $(\widetilde{G}, \widetilde{H})$ является $(K, T)$-максимальной в графе $\Gamma$, если в графе $\Gamma \backslash \widetilde{G}$ не существует вершины, соединенной с ровно двумя из $\tilde{x}_{1}, \tilde{x}_{2}, \tilde{x}_{3}$. Заметим, что в нашем примере пара $(\widetilde{G}, \widetilde{H})$ является $1 / 2$-надежной, а пара $(K, T)$ является $1 / 2$-нейтральной. Полученные нами результаты, которые будут сформулированы в следующем разделе, справедливы именно для $(K, T)$-максимальных $\alpha$-надежных пар, где пара $(K, T)$ является $\alpha$-нейтральной.

\section{2. Формулировки новых результатов}

Рассмотрим снова случайный граф

$$
G(N, p(N))=\left(\Omega_{N}, \mathscr{F}_{N}, \mathbf{P}_{N, p}\right)
$$

и два графа $H$ и $G$ таких, что $H \subset G, V(H)=\left\{x_{1}, \ldots, x_{k}\right\}, V(G)=\left\{x_{1}, \ldots, x_{l}\right\}$. Пусть $\tilde{x}_{1}, \ldots, \tilde{x}_{k} \in \mathscr{V}$ и случайная величина $N_{(G, H)}^{(K, T)}\left(\tilde{x}_{1}, \ldots, \tilde{x}_{k}\right)$ на вероятностном пространстве $G(N, p(N))$ каждому графу $\mathscr{G}$ из $\Omega_{N}$ ставит в соответствие число таких $(G, H)$ расширений $\widetilde{G}$ подграфа в $\mathscr{G}$, индуцированного на $\left\{\tilde{x}_{1}, \ldots, \tilde{x}_{k}\right\}$, что пара $\left(\widetilde{G},\left.\mathscr{G}\right|_{\left\{\tilde{x}_{1}, \ldots, \tilde{x}_{k}\right\}}\right)$ 
является $(K, T)$-максимальной в $\mathscr{G}$. Иными словами, пусть $W \subset \mathscr{V} \backslash\left\{\tilde{x}_{1}, \ldots, \tilde{x}_{k}\right\}-$ множество, мощность $|W|$ которого равна $l-k$. Если можно так занумеровать элементы множества $W$ числами $k+1, k+2, \ldots, l$, что граф $G_{\left\{\tilde{x}_{1}, \ldots, \tilde{x}_{l}\right\}}$ является $(G, H)$-расширением графа $\left.\mathscr{G}\right|_{\left\{\tilde{x}_{1}, \ldots, \tilde{x}_{k}\right\}}$ и пара $\left(\left.\mathscr{G}\right|_{\left\{\tilde{x}_{1}, \ldots, \tilde{x}_{l}\right\}},\left.\mathscr{G}\right|_{\left\{\tilde{x}_{1}, \ldots, \tilde{x}_{k}\right\}}\right)$ является $(K, T)$-максимальной, то положим $I_{W}(\mathscr{G})=1$. В противном случае положим $I_{W}(\mathscr{G})=0$. Случайная величина $N_{(G, H)}^{(K, T)}\left(\tilde{x}_{1}, \ldots, \tilde{x}_{k}\right)$ определяется равенством

$$
N_{(G, H)}^{(K, T)}\left(\tilde{x}_{1}, \ldots, \tilde{x}_{k}\right)=\sum_{\substack{W \subset \mathcal{V} \backslash\left\{\tilde{x}_{1}, \ldots, \tilde{x}_{k}\right\} \\|W|=l-k}} I_{W} .
$$

Введем, кроме того, случайную величину $N_{(G, H)}^{\left(K_{1}, T_{1}\right),\left(K_{2}, T_{2}\right), \ldots,\left(K_{s}, T_{S}\right)}\left(\tilde{x}_{1}, \ldots, \tilde{x}_{k}\right)$, которая каждому графу $\mathscr{G}$ из $\Omega_{N}$ ставит в соответствие число таких $(G, H)$-расширений $\widetilde{G}$ подграфа в $\mathscr{G}$, индуцированного на $\left\{\tilde{x}_{1}, \ldots, \tilde{x}_{k}\right\}$, что пара $\left(\widetilde{G},\left.\mathscr{G}\right|_{\left\{\tilde{x}_{1}, \ldots, \tilde{x}_{k}\right\}}\right)$ является $\left(K_{1}, T_{1}\right)$ максимальной, $\left(K_{2}, T_{2}\right)$-максимальной, .., $\left(K_{s}, T_{s}\right)$-максимальной в $\mathscr{G}$.

Пусть пара $(G, H)$ является $\alpha$-надежной и $p=N^{-\alpha}$. Из результатов, полученных Дж. Спенсером (см. $[1,2,5])$, следуют оценки величин $N_{(G, H)}^{(K, T)}\left(\tilde{x}_{1}, \ldots, \tilde{x}_{k}\right)$, если пара $(K, T)$ является $\alpha$-надежной. А именно, с асимптотической вероятностью 1 для любых $\tilde{x}_{1}, \ldots, \tilde{x}_{k}$ справедливо равенство $N_{(G, H)}^{(K, T)}\left(\tilde{x}_{1}, \ldots, \tilde{x}_{k}\right)=0$. Если же пара $(K, T)$ является $\alpha$-жесткой и число $\alpha$ иррационально, то оценки для этих случайных величин выводятся из тех же результатов (см. лемму 10.7.6 в [2]); если $\alpha$ рационально, то оценки следуют из доказанной нами в настоящей работе леммы о нейтральных расширениях и леммы 10.7.6 в [2]. А именно, с асимптотической вероятностью 1 для любых $\tilde{x}_{1}, \ldots, \tilde{x}_{k}$ справедливо соотношение

$$
N_{(G, H)}^{(K, T)}\left(\tilde{x}_{1}, \ldots, \tilde{x}_{k}\right) \sim N_{(G, H)}\left(\tilde{x}_{1}, \ldots, \tilde{x}_{k}\right) .
$$

Этот результат непосредственно вытекает из описанных лемм, поэтому не обсуждается.

Теорема 2. Пусть пары $\left(K_{1}, T_{1}\right),\left(K_{2}, T_{2}\right), \ldots,\left(K_{s}, T_{s}\right)$ являются $\alpha$-нейтральными. При сделанных предположениях, с асимптотической вероятностью 1 для любых вершин $\tilde{x}_{1}, \ldots, \tilde{x}_{k}$

$$
\begin{aligned}
N_{(G, H)}^{\left(K_{1}, T_{1}\right),\left(K_{2}, T_{2}\right), \ldots,\left(K_{S}, T_{S}\right)}\left(\tilde{x}_{1}, \ldots, \tilde{x}_{k}\right) & \sim \mathbf{E}_{N, p} N_{(G, H)}^{\left(K_{1}, T_{1}\right),\left(K_{2}, T_{2}\right), \ldots,\left(K_{S}, T_{s}\right)}\left(\tilde{x}_{1}, \ldots, \tilde{x}_{k}\right) \\
& =\Theta\left(N^{v(G, H)-\alpha e(G, H)}\right) .
\end{aligned}
$$

Прежде чем обосновать справедливость этого результата, мы докажем его частный случай для одной $\alpha$-нейтральной пары, который мы формулируем здесь в виде отдельного утверждения.

Предложение 1. Пусть $p=N^{-\alpha}$, где $0<\alpha \leqslant 1-$ раџиональное число, пара $(G, H)$ является $\alpha$-надежной, а пара $(K, T)$ является $\alpha$-нейтральной. Тогда с асимптотической вероятностью 1 для любых вершин $\tilde{x}_{1}, \ldots, \tilde{x}_{k}$

$$
N_{(G, H)}^{(K, T)}\left(\tilde{x}_{1}, \ldots, \tilde{x}_{k}\right) \sim \mathbf{E}_{N, p} N_{(G, H)}^{(K, T)}\left(\tilde{x}_{1}, \ldots, \tilde{x}_{k}\right)=\Theta\left(N^{v(G, H)-\alpha e(G, H)}\right) .
$$

\section{3. Доказательство основных результатов}

\section{1. Лемма о композиции нейтральной и надежной пары}

Лемма 1. Пусть пара $(G, H)$ является $\alpha$-надежной, $T-$ подграф графа $G$, причем $T \cap H \neq T$; пара $(K, T)$ является $\alpha$-нейтральной, $K \cap G=T$, причем множество 


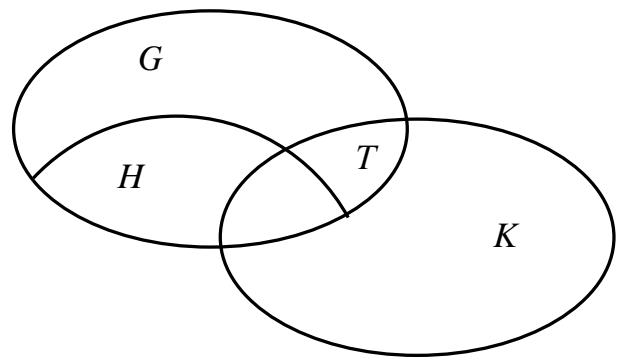

Pис. 2.

$E((K \backslash T) \cup(G \backslash T)) \backslash(E(K \backslash T) \cup E(G \backslash T))=\varnothing$. При сделанных предположениях пара $(K \cup H, H)$ является $\alpha$-надежной.

Доказательство. Пусть граф $S$ таков, что

$$
H \subset S \subset K \cup H, \quad V(S)=V(H) \cup\left\{x_{1}, \ldots, x_{m}, y_{1}, \ldots, y_{n}\right\},
$$

именно, $x_{1}, \ldots, x_{m} \in V(G) \backslash V(H)$ и $y_{1}, \ldots, y_{n} \in V(K) \backslash V(G)$. Тогда в силу того, что $(G, H)-\alpha$-надежная пара, суммарное число ребер между вершинами $x_{1}, \ldots, x_{m}$ и ребер между вершинами $x_{1}, \ldots, x_{m}$ и вершинами графа $H$ меньше, чем $m / \alpha$.

Далее, если $m \neq 0$, то ввиду $\alpha$-нейтральности пары $(K, T)$ суммарное число ребер между вершинами $x_{1}, \ldots, x_{m}$ и вершинами $y_{1}, \ldots, y_{n}$, ребер между вершинами $y_{1}, \ldots, y_{n}$ и ребер между вершинами $y_{1}, \ldots, y_{n}$ и вершинами графа $H$ не больше, чем $n / \alpha$. Если же $m=0$, то суммарное число ребер между вершинами $y_{1}, \ldots, y_{n}$ и ребер между вершинами $y_{1}, \ldots, y_{n}$ и вершинами графа $H$ меньше, чем $n / \alpha$, так как по условию $T \cap H \neq T$, а следовательно, пара $(K \backslash(G \backslash H), T \cap H) \alpha$-надежная. Таким образом,

$$
v(S, H)-\alpha e(S, H)=(n+m)-\alpha e(S, H)>(n+m)-\alpha n+m / \alpha=0 .
$$

Поэтому пара $(K \cup H, H)$ является $\alpha$-надежной. Лемма доказана.

Заметим, что в теореме 1 говорится не о точных расширениях. Для доказательства наших результатов придется рассмотреть точные расширения и оценить их число. Тем самым, потребуется усилить утверждение теоремы 1. Иными словами, мы покажем, что число расширений с дополнительным ребром бесконечно мало по сравнению с числом точных расширений. В работах [2] и [3] это доказано для случая иррационального $\alpha$, мы же докажем это для всех $\alpha \in(0,1]$.

\section{2. Теорема о точных надежных расширениях}

Рассмотрим случайный граф

$$
G(N, p(N))=\left(\Omega_{N}, \mathscr{F}_{N}, \mathbf{P}_{N, p}\right)
$$

и два графа $H$ и $G, H \subset G$. Пусть $V(H)=\left\{x_{1}, \ldots, x_{k}\right\}, V(G)=\left\{x_{1}, \ldots, x_{l}\right\}$ и $\tilde{x}_{1}, \ldots, \tilde{x}_{k} \in \mathscr{V}$. Пусть $N_{(G, H)}^{\text {strict }}\left(\tilde{x}_{1}, \ldots, \tilde{x}_{k}\right)$ - число точных $(G, H)$-расширений множества $\left\{\tilde{x}_{1}, \ldots, \tilde{x}_{k}\right\}$, то есть случайная величина $N_{(G, H)}^{\text {strict }}\left(\tilde{x}_{1}, \ldots, \tilde{x}_{k}\right)$ каждому графу $\mathscr{G}$ из $\Omega_{N}$ ставит в соответствие число точных $(G, H)$-расширений подграфа в $\mathscr{G}$, индуцированного на $\left\{\tilde{x}_{1}, \ldots, \tilde{x}_{k}\right\}$ (мы не даем здесь более формального определения, поскольку оно аналогично определению случайной величины $\left.N_{(G, H)}\left(\tilde{x}_{1}, \ldots, \tilde{x}_{k}\right)\right)$. 
Теорема 3. Пусть $p=N^{-\alpha}$, где $0<\alpha \leqslant 1, u(G, H)-\alpha$-надежная пара. Тогда с асимптотической вероятностью 1 для любых вершин $\tilde{x}_{1}, \ldots, \tilde{x}_{k}$ справедливо соотношение

$$
N_{(G, H)}^{\text {strict }}\left(\tilde{x}_{1}, \ldots, \tilde{x}_{k}\right) \sim N_{(G, H)}\left(\tilde{x}_{1}, \ldots, \tilde{x}_{k}\right) .
$$

Для доказательства этого результата нам потребуется ввести некоторые определения, а также доказать ряд лемм.

\subsection{1. Жесткие и нейтральные цепи. Сбалансированные графы. Вспомогательные утверждения}

Напомним определение жесткой цепи, введенное в $[2,3])$. Рассмотрим графы $H, G$, $H \subset G$. Если существуют такие графы $S_{0}, S_{1}, \ldots, S_{r}$, что $H=S_{0} \subset S_{1} \subset \ldots \subset S_{r}=G$, пары $\left(S_{i}, S_{i-1}\right)$ являются $\alpha$-жесткими и для некоторого $t$ при любом $i \in\{1,2, \ldots, r\}$ выполнено неравенство $\left|V\left(S_{i}\right) \backslash V\left(S_{i-1}\right)\right| \leqslant t$, то пара $(G, H)$ называется $\alpha$-жесткой $t$-цепью. Если $X \subset Y \subset \Gamma$ и пара $(Y, X)$ является $\alpha$-жесткой $t$-цепью и для нее не существует такого графа $Z \subset \Gamma$, что пара $(Z, Y)$ является $\alpha$-жесткой, $|V(Z) \backslash V(Y)| \leqslant t$, то пара $(Y, X)$ называется максимальной в $\Gamma \alpha$-жесткой $t$-цепью, а граф $Y$ обозначается $c l_{t}(X)$. У графа $X$ существует только один такой граф $Y$ в $\Gamma$, что пара $(Y, X)$ является максимальной в $\Gamma \alpha$-жесткой $t$-цепью. Действительно, если пары $(Y, X),(Z, X)$ являются $\alpha$-жесткими $t$-цепями, то пара $(Y \cup Z, X)$ тоже является $\alpha$-жесткой $t$-цепью.

Введем понятие нейтральной цепи. Будем говорить, что пара $(G, H)$ является $\alpha$-нейтральной цепью, если найдутся такие графы $K_{1}, \ldots, K_{r}$ и $T_{1}, \ldots, T_{r-1}$, что $H=K_{1} \subset K_{2} \subset \ldots \subset K_{r}=G$, для каждого $i \in\{1, \ldots, r-1\}$ справедливо включение $T_{i} \subseteq K_{i}$, пара $\left(\left(K_{i+1} \backslash K_{i}\right) \cup T_{i}, T_{i}\right)$ является $\alpha$-нейтральной, и между графами $K_{i+1} \backslash K_{i}$ и $K_{i} \backslash T_{i}$ нет ребер.

Сформулируем теперь определения сбалансированных и строго сбалансированных графов, которые рассматривали П. Эрдеш и А. Реньи (см. [11]). Пусть $G$ - граф с $v$ вершинами и $е$ ребрами. Отношение $\rho(G)=e / v$ называется плотностью графа $G$. Положим

$$
\rho^{\max }(G)=\max \{\rho(H): H \subseteq G\} .
$$

Граф $G$ называется сбалансированным, если для каждого его подграфа $H$ выполнено неравенство $\rho(H) \leqslant \rho(G)$. Граф $G$ называется строго сбалансированным, если для любого $H \subset G$ справедливо строгое неравенство $\rho(H)<\rho(G)$. Сформулируем теперь теорему о числе копий строго сбалансированного графа.

Теорема 4 ([2, 4]). Пусть $G(N, p)$ есть случайный граф, $G$ есть произвольный граф, $N_{G}$ есть число копий $G$ в случайном графе. Тогда функциия $p=N^{-1 / \rho^{\max }(G)}$ является пороговой для свойства содержать копию графа $G$. Если граф $G$ сбалансированньй, то для любого $\beta>0$ с асимптотической вероятностью 1 справедливо равенство $N_{G}=o\left(N^{\beta}\right)$. Если же граф $G$ строго сбалансированный и $p=\omega\left(N^{-1 / \rho(G)}\right)$, mо $N_{G} \sim \mathbf{E}_{N, p} N_{G} c$ асимптотической вероятностью 1.

Пусть $S_{1}, S_{2}$ - два графа, причем один из них является подграфом другого. Рассмотрим функцию $f$, определенную на парах вложенных друг в друга графов. Если $S_{2} \subseteq S_{1}$, то определим значение функции $f$ на паре $\left(S_{1}, S_{2}\right)$ равенством $f\left(S_{1}, S_{2}\right)=$ $v\left(S_{1}, S_{2}\right)-\alpha e\left(S_{1}, S_{2}\right)$. Если же $S_{1} \subseteq S_{2}$, то $f\left(S_{1}, S_{2}\right)=-v\left(S_{2}, S_{1}\right)+\alpha e\left(S_{2}, S_{1}\right)$. Иными словами, если $H-$ такой граф, что $H \subseteq S_{1}, H \subseteq S_{2}$, то

$$
f\left(S_{1}, S_{2}\right)=\left(v\left(S_{1}, H\right)-v\left(S_{2}, H\right)\right)-\alpha\left(e\left(S_{1}, H\right)-e\left(S_{2}, H\right)\right) .
$$


Заметим, что справедливо равенство

$$
f\left(S_{1}, S_{2}\right)-f\left(S_{1}, S_{3}\right)=f\left(S_{3}, S_{2}\right) .
$$

Докажем вспомогательную лемму об устройстве любой пары $(G, H)$.

Лемма 2. Пусть $H \subset G$, причем для любого такого графа $S, S \subseteq G \backslash H$, что множество ребер $E(G) \backslash(E(G \backslash S) \cup E(S))$ пусто, справедливо неравенство $f(G, G \backslash S) \neq 0$. Тогда выполнено одно из двух свойств:

- найдется такой граф $X$, что $H \subseteq X \subseteq G$ и пары $(X, H)$ и $(G, X)$ являются $\alpha$-нейтральной ичепью и $\alpha$-надежной ичепью соответственно;

- найдутся такие графыл $X, Y$, что $H \subset Y \subseteq X \subseteq G$ и napbl $(Y, H),(X, Y) u(G, X)$ являются $\alpha$-жесткой, $\alpha$-нейтральной и $\alpha$-надежной цеепью соответственно.

Доказательство. Если для любого такого $S$, что $H \subset S \subseteq G$, справедливо неравенство $f(S, H) \geqslant 0$, то выполнено первое свойство. Действительно, при наших предположениях найдутся такие графы $S_{0}, \ldots, S_{s}$, что $H=S_{0} \subset S_{1} \subset S_{2} \subset \ldots \subset S_{s}=G$ и для любого $i \in\{1,2, \ldots, s-1\}$ справедливо равенство $f\left(S_{i}, H\right)=0$, причем $E\left(S_{i}\right) \backslash\left(E\left(S_{i-1}\right) \cup E\left(S_{i} \backslash S_{i-1}\right)\right) \neq \varnothing$, для любого такого $S$, что $S_{i-1} \subset S \subset S_{i}$, выполнено неравенство $f(S, H)>0$, для любого такого $S$, что $S_{S-1} \subset S \subset S_{S}$, также выполнено неравенство $f(S, H)>0$, при этом либо $f\left(S_{S}, H\right)>0$, либо $f\left(S_{S}, H\right)=0$ и $E\left(S_{S}\right) \backslash\left(E\left(S_{S-1}\right) \cup E\left(S_{S} \backslash S_{S-1}\right)\right) \neq \varnothing$. Следовательно, в силу равенства (1) для любого $i \in\{1,2, \ldots, s-1\}$ справедливо равенство $f\left(S_{i}, S_{i-1}\right)=0$; для любого такого $S$, что $S_{i-1} \subset S \subset S_{i}$, выполнено неравенство $f\left(S, S_{i-1}\right)>0$; для любого такого $S$, что $S_{S-1} \subset S \subset S_{s}$, также выполнено неравенство $f\left(S, S_{s-1}\right)>0$, при этом либо $f\left(S_{s}, S_{s-1}\right)>0$, либо $f\left(S_{s}, S_{s-1}\right)=0$. Если справедливо $f\left(S_{s}, S_{s-1}\right)>0$, то пара $\left(S_{s-1}, H\right)$ является $\alpha$-нейтральной цепью, а пара $\left(G, S_{s-1}\right)$ является $\alpha$-надежной цепью. Действительно, для каждого $i \in\{1,2, \ldots, s-1\}$ мы можем выбрать в графе $S_{i-1}$ непустой подграф $T_{i-1}$, каждая вершина которого соединена с некоторой вершиной графа $S_{i} \backslash S_{i-1}$ (так как $\left.E\left(S_{i}\right) \backslash\left(E\left(S_{i-1}\right) \cup E\left(S_{i} \backslash S_{i-1}\right)\right) \neq \varnothing\right)$, тогда пара $\left(S_{i} \backslash\left(S_{i-1} \backslash T_{i-1}\right), T_{i-1}\right)$ является $\alpha$-нейтральной. Во втором случае (где $\left.f\left(S_{S}, S_{s-1}\right)=0\right)$ пара $(G, H)$ является $\alpha$-нейтральной цепью. Итак, первая часть леммы верна.

Пусть теперь $S$ - такой граф, что $H \subset S \subseteq G$ и $f(S, H)<0$. Покажем, что найдется такой граф $\widehat{S}_{1}$, что $H \subset \widehat{S}_{1} \subseteq S$ и пара $\left(\widehat{S}_{1}, H\right)$ является $\alpha$-жесткой.

Если для любого такого графа $S_{0}$, что $H \subset S_{0} \subset S$, верно неравенство $f\left(S, S_{0}\right)<0$, то пара $(S, H)$ является $\alpha$-жесткой.

Предположим, что нашлось ровно $r$ таких графов $S_{1}, \ldots, S_{r}$, что $H \subset S_{i} \subset S$ и справедливо неравенство $f\left(S, S_{i}\right) \geqslant 0$. Тогда найдется такой граф $S_{j}$, что $j \in\{1,2, \ldots, r\}$ и для любого $i \in\{1,2, \ldots, r\}$, отличного от $j$, выполнено неравенство $S_{j} \cap S_{i} \neq S_{j}$. Значит, для любого такого графа $\widetilde{T}$, что $H \subseteq \widetilde{T} \subset S_{j}$, справедливо неравенство $f(S, \widetilde{T})<0$, а следовательно, в силу тождества (1) выполнено неравенство $f\left(S_{j}, \widetilde{T}\right)<0$, то есть пара $\left(S_{j}, H\right)$ является $\alpha$-жесткой. Таким образом, мы нашли такой граф $\widehat{S}_{1}$, что пара $\left(\widehat{S}_{1}, H\right)$ является $\alpha$-жесткой.

Рассмотрим теперь пару $\left(G, \widehat{S}_{1}\right)$, применим для нее те же рассуждения, то есть найдем такой граф $\widehat{S}_{2}$, что пара $\left(\widehat{S}_{2}, \widehat{S}_{1}\right)$ является $\alpha$-жесткой, и так до тех пор, пока среди подграфов графа $G$, являющихся расширением графа $\widehat{S}_{S}$, не найдется такого, что $f\left(\cdot, \widehat{S}_{S}\right)<0$. Заметим, что если пары $(B, A)$ и $(C, B)$ является $\alpha$-жесткими, то пара $(C, A)$ также является $\alpha$-жесткой. Таким образом, пара $\left(\widehat{S}_{s}, H\right)$ является $\alpha$-жесткой.

Лемма доказана. 
Сформулируем теперь лемму о числе надежных расширений.

Лемма 3. Пусть $p=N^{-\alpha}, \alpha>0$. Пусть, кроме того, пара $(G, H)$ является $\alpha$-надежной, $H \subset S \subset G$, и пара $(G, S)$ является $\alpha$-надежной. Если $|V(H)|=k,|V(S)|=s$, то сущзествует такое число $\beta>0$, что с асимптотической вероятностью 1 для любых вершин $\tilde{x}_{1}, \ldots, \tilde{x}_{k}, \tilde{y}_{1}, \ldots, \tilde{y}_{s}$ справедливо равенство

$$
N_{(G, S)}\left(\tilde{y}_{1}, \ldots, \tilde{y}_{S}\right)=\Theta\left(N_{(G, H)}\left(\tilde{x}_{1}, \ldots, \tilde{x}_{k}\right) N^{-\beta}\right) .
$$

Доказательство. В силу того, что пара $(G, H)$ является $\alpha$-надежной, выполнено неравенство $f(S, H)>0$. Поэтому, в силу тождества (1), справедливо неравенство $f(G, S)<f(G, H)$. Положим

$$
\beta=f(G, H)-f(G, S) .
$$

Обратимся к теореме 1. Из нее, очевидно, следует, что с асимптотической вероятностью 1 для любых вершин $\tilde{x}_{1}, \ldots, \tilde{x}_{k}, \tilde{y}_{1}, \ldots, \tilde{y}_{s}$ справедливы соотношения

$$
\begin{gathered}
N_{(G, H)}\left(\tilde{x}_{1}, \ldots, \tilde{x}_{k}\right)=\Theta\left(N^{f(G, H)}\right), \\
N_{(G, S)}\left(\tilde{y}_{1}, \ldots, \tilde{y}_{S}\right)=\Theta\left(N^{f(G, S)}\right) .
\end{gathered}
$$

Следовательно,

$$
N_{(G, S)}\left(\tilde{y}_{1}, \ldots, \tilde{y}_{S}\right)=\Theta\left(N_{(G, H)}\left(\tilde{x}_{1}, \ldots, \tilde{x}_{k}\right) N^{-\beta}\right) .
$$

Лемма доказана.

В 1988 г. С. Шела и Дж. Спенсер доказали сформулированную ниже лемму о жестких цепях.

Лемма 4 ([2, 3]). Пусть $p=N^{-\alpha}, \alpha>0$. Пусть, кроме того, $t, r-$ произвольные натуральные числа. Тогда существует такое число $K(t, r)$, что с асимптотической вероятностью 1 для любых вершин $\tilde{x}_{1}, \ldots, \tilde{x}_{r}$ выполнено неравенство

$$
\left|V\left(c l_{t}\left(\tilde{x}_{1}, \ldots, \tilde{x}_{r}\right)\right)\right| \leqslant K(t, r) .
$$

Сформулируем, наконец, лемму о числе нейтральных расширений.

Лемма 5. Пусть

$$
p=N^{-\alpha}, \quad 0<\alpha \leqslant 1, \quad \beta>0 .
$$

Пусть, кроме того, пара $(G, H)$ является $\alpha$-нейтральной. Если $|V(H)|=k$, то с асимптотической вероятностью 1 для любых вершин $\tilde{x}_{1}, \ldots, \tilde{x}_{k}$ справедливы соотношения

$$
\begin{aligned}
& N_{(G, H)}\left(\tilde{x}_{1}, \ldots, \tilde{x}_{k}\right)=o\left(N^{\beta}\right), \\
& N_{(G, H)}^{\text {strict }}\left(\tilde{x}_{1}, \ldots, \tilde{x}_{k}\right)=o\left(N^{\beta}\right) .
\end{aligned}
$$

Доказательство. Покажем, что если утверждение теоремы не выполнено, то в графе с асимптотической вероятностью 1 найдется огромное число копий строго сбалансированного графа, являющихся расширениями одного и того же подграфа. Это число окажется асимптотически бо́льшим, чем то, которое возникает в теореме 4. Напомним, что мы 
считаем справедливым равенство $|V(G)|=l$. Пусть $\xi \in \mathbf{N}$ таково, что справедливо неравенство

$$
\xi>\max \left\{\frac{2 k^{2}(l-k)}{\alpha}, \frac{2 k}{\beta}\right\} .
$$

Рассмотрим $R_{1}, \ldots, R_{\xi}$ - точные $(G, H)$-расширения графа $H$, каждые два из которых пересекаются в точности по $H$. Пусть между любыми двумя из этих расширений нет ребер, то есть для любых $i \neq j$ множество $E\left(R_{i} \cup R_{j} \backslash H\right) \backslash\left(E\left(R_{i} \backslash H\right) \cup E\left(R_{j} \backslash H\right)\right)$ пусто. Положим $S=R_{1} \cup \ldots \cup R_{\xi}$. Будем считать, что $E(H)=\varnothing$. Справедливость всех дальнейших выводов от этого не изменится, потому что в графе $H$ нам важен только порядок и число вершин. Докажем, что при сделанном предположении граф $S$ является строго сбалансированным с плотностью

$$
\rho(S)=\frac{\xi(l-k)}{\alpha(\xi(l-k)+k)} .
$$

Рассмотрим некоторый подграф $X$ в $S$ и его пересечения с графами $R_{1} \backslash H, R_{2} \backslash H, \ldots$, $R_{\xi} \backslash H$. Предположим, что он имеет ненулевое пересечение только с графами $R_{i_{1}} \backslash H, \ldots$, $R_{i_{v}} \backslash H$. Пусть $X_{0}=H \cap X, X_{1}=\left(R_{i_{1}} \backslash H\right) \cap X, X_{2}=\left(R_{i_{2}} \backslash H\right) \cap X, \ldots, X_{v}=\left(R_{i_{v}} \backslash H\right) \cap X$. Пусть, кроме того, $v_{0}=\left|V\left(X_{0}\right)\right|, v_{1}=\left|V\left(X_{1}\right)\right|, \ldots, v_{v}=\left|V\left(X_{v}\right)\right|, e_{0}=\left|E\left(X_{0}\right)\right|=0$, $e_{1}=\left|E\left(X_{1} \cup X_{0}\right) \backslash E\left(X_{0}\right)\right|=\left|E\left(X_{1} \cup X_{0}\right)\right|, e_{2}=\left|E\left(X_{2} \cup X_{0}\right) \backslash E\left(X_{0}\right)\right|=\left|E\left(X_{2} \cup X_{0}\right)\right|, \ldots$, $e_{v}=\left|E\left(X_{v} \cup X_{0}\right) \backslash E\left(X_{0}\right)\right|=\left|E\left(X_{v} \cup X_{0}\right)\right|$.

Справедливо равенство

$$
\rho(X)=\frac{\sum_{i=0}^{v} e_{i}}{\sum_{i=0}^{v} v_{i}}
$$

Рассмотрим два случая: первый $-X_{0}=H$, второй $-X_{0} \subset H$. Оценим $\rho(X)$ в обоих случаях. Обратимся к первому случаю. Справедливы соотношения

$$
\rho(X)=\frac{\sum_{i=1}^{v} e_{i}}{\sum_{i=1}^{v} v_{i}+v_{0}} \leqslant \frac{\sum_{i=1}^{v} v_{i}}{\alpha\left(\sum_{i=1}^{v} v_{i}+k\right)}
$$

Следовательно, $\rho(X)<\rho(S)$ при $\sum_{i=1}^{v} v_{i}<\xi(l-k)$ (в этом случае $\left.X \subset S\right)$. Если $\sum_{i=1}^{v} v_{i}=\xi(l-k)$, то $X=S$ и $\rho(X)=\rho(S)$.

Рассмотрим второй случай: $X_{0} \subset H$. Разобьем множество $I=\{1,2, \ldots, v\}$ на два подмножества $I_{1}, I_{2}, I=I_{1} \sqcup I_{2}$ в соответствие со следующими условиями:

$$
i \in I_{1} \Longleftrightarrow v_{i}=l-k, \quad i \in I_{2} \Longleftrightarrow v_{i}<l-k .
$$

Если $i \in I_{1}$, то

$$
e_{i}=e\left((G \backslash H) \cup X_{0}, X_{0}\right) \leqslant e(G, H)-\left(k-v_{0}\right)=\frac{v_{i}}{\alpha}-\left(k-v_{0}\right)
$$


(неравенство в этой цепочке справедливо, так как по определению нейтральной пары каждая вершина из $H \backslash X_{0}$ соединена с некоторой вершиной из $\left.X_{i}\right)$. Если же $i \in I_{2}$, то

$$
\begin{gathered}
e_{i}<e\left(X_{i} \cup H, H\right)<\frac{v_{i}}{\alpha}, \\
e_{i} \leqslant v_{i} / \alpha-\frac{1}{v(G, H)}=\frac{v_{i}}{\alpha}-\frac{1}{l-k}
\end{gathered}
$$

(последнее неравенство выполнено, так как $\alpha=v(G, H) / e(G, H))$. В силу сделанных выводов

$$
\rho(X)=\frac{\sum_{i \in I_{1}} e_{i}+\sum_{i \in I_{2}} e_{i}}{\sum_{i=1}^{v} v_{i}+v_{0}} \leqslant \frac{\sum_{i=1}^{v} v_{i}-\alpha\left(\left|I_{1}\right|\left(k-v_{0}\right)+\frac{\left|I_{2}\right|}{l-k}\right)}{\alpha\left(\sum_{i=1}^{v} v_{i}+v_{0}\right)} .
$$

Предположим, что

$$
\sum_{i=1}^{v} v_{i}<\frac{v_{0} \xi(l-k)}{k}
$$

При таком допущении

$$
\rho(X) \leqslant \frac{\sum_{i=1}^{v} v_{i}}{\alpha\left(\sum_{i=1}^{v} v_{i}+v_{0}\right)}<\frac{v_{0} \xi(l-k)}{k \alpha\left(v_{0} \xi(l-k) / k+v_{0}\right)}=\rho(S) .
$$

Предположим теперь, что

$$
\sum_{i=1}^{v} v_{i} \geqslant \frac{v_{0} \xi(l-k)}{k}, \quad v_{0} \neq 0 .
$$

Тогда справедливо неравенство

$$
|I| \geqslant \frac{v_{0} \xi}{k}
$$

а следовательно,

$$
\max \left\{\left|I_{1}\right|,\left|I_{2}\right|\right\} \geqslant \frac{v_{0} \xi}{2 k} .
$$

Поэтому выполнены соотношения

$$
\begin{gathered}
\rho(X) \leqslant \frac{\sum_{i=1}^{v} v_{i}-\frac{\alpha v_{0} \xi}{2 k} \min \left\{k-v_{0}, \frac{1}{l-k}\right\}}{\alpha\left(\sum_{i=1}^{v} v_{i}+v_{0}\right)} \\
\leqslant \frac{1}{\alpha}-\frac{\xi}{2(l-k)(\xi(l-k)+k) k}<\rho(S) .
\end{gathered}
$$


Последнее неравенство справедливо в силу выбора $\xi$.

Осталось рассмотреть случай $v_{0}=0$. Воспользовавшись неравенством

$$
\max \left\{\left|I_{1}\right|,\left|I_{2}\right|\right\} \geqslant \frac{v}{2},
$$

получим, что

$$
\begin{aligned}
\rho(X) \leqslant & \frac{\sum_{i=1}^{v} v_{i}-\frac{\alpha \nu}{2(l-k)}}{\alpha \sum_{i=1}^{v} v_{i}} \leqslant \frac{\nu(l-k)-\alpha v /(2(l-k))}{\alpha \nu(l-k)} \\
= & \frac{1}{\alpha}-\frac{1}{2(l-k)^{2}}<\rho(S),
\end{aligned}
$$

где последнее неравенство справедливо опять благодаря выбору $\xi$. Итак, $S-$ строго сбалансированный граф с плотностью

$$
\rho(S)=\frac{\xi(l-k)}{\alpha(\xi(l-k)+k)}<\frac{1}{\alpha} .
$$

Рассмотрим событие $\mathscr{A}_{\beta}\left(\tilde{x}_{1}, \ldots, \tilde{x}_{k}\right)$, заключающееся в том, что у вершин $\tilde{x}_{1}, \ldots, \tilde{x}_{k}$ найдется не менее $N^{\beta / 2}$ точных $(G, H)$-расширений. Пусть $\mathscr{S}_{0} \subseteq \Omega_{N}-$ множество графов, у которых существуют такие вершины $\tilde{x}_{1}, \ldots, \tilde{x}_{k}$, что выполняется свойство $\mathscr{A}_{\beta}\left(\tilde{x}_{1}, \ldots, \tilde{x}_{k}\right)$. В дальнейшем нам потребуется несколько раз применять лемму о жестких цепях. Поэтому рассмотрим множество графов $\mathscr{S}_{1} \subseteq \Omega_{N}$, для которых утверждение леммы о жестких цепях верно при $t=2(l-k), r \in\{k, k+1, \ldots, l\}$. Иными словами, для каждого графа $\widetilde{G}_{1} \in \mathscr{S}_{1}$ и для любого $r \in\{k, k+1, \ldots, l\}$ существует такая константа $K(2(l-k), r)$, что для любых вершин $\tilde{x}_{1}, \ldots, \tilde{x}_{r}$ графа $\widetilde{G}_{1}$ справедливо неравенство

$$
\left|V\left(c l_{2(l-k)}\left(\tilde{x}_{1}, \ldots, \tilde{x}_{r}\right)\right)\right| \leqslant K(2(l-k), r) .
$$

Пусть $\mathscr{S}_{2} \subseteq \Omega_{N}-$ множество графов, в которых число копий $S$ ограничено сверху величиной $\Theta\left(N^{(\xi(l-k)+k)(1-\alpha \rho(S))}\right)=\Theta\left(N^{(\xi(l-k)+k)(1-\xi(l-k)(\xi(l-k)+k)}\right)=\Theta\left(N^{k}\right)$. Положим $\mathscr{S}=\mathscr{Y}_{0} \cap \mathscr{Y}_{1} \cap \mathscr{Y}_{2}$. Требуется доказать справедливость равенства

$$
\lim _{N \rightarrow \infty} \mathbf{P}_{N, p}\left(\mathscr{S}_{0}\right)=0 \text {. }
$$

В силу леммы о жестких цепях и теоремы 4, справедливо тождество

$$
\lim _{N \rightarrow \infty} \mathbf{P}_{N, p}\left(\mathscr{S}_{1} \cap \mathscr{S}_{2}\right)=1
$$

Поэтому достаточно показать, что вероятность $\mathbf{P}_{N, p}(\mathscr{Y})$ стремится к нулю при $N \rightarrow \infty$.

Пусть $\widetilde{G} \in \underset{\mathscr{T}}{\mathscr{S}}$. Следовательно, $\widetilde{G} \in \mathscr{Y}_{0}$, иными словами, существуют вершины $\tilde{x}_{1}, \ldots, \tilde{x}_{k} \in V(\widetilde{G})$, для которых граф $\widetilde{G}$ обладает свойством $\mathscr{A}_{\beta}\left(\tilde{x}_{1}, \ldots, \tilde{x}_{k}\right)$. Зафиксируем $\tilde{x}_{1}, \ldots, \tilde{x}_{k}$. Покажем, что в графе $\widetilde{G}$ существуют точные $(G, H)$-расширения

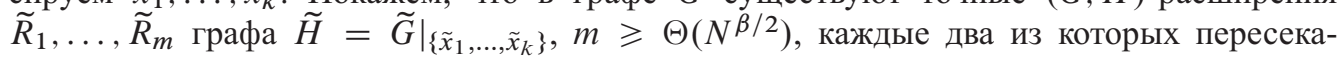
ются в точности по $\tilde{H}$, обладающие следующим свойством: для любых $i \neq j$ множество $E\left(\widetilde{R}_{i} \cup \widetilde{R}_{j} \backslash \widetilde{H}\right) \backslash\left(E\left(\widetilde{R}_{i} \backslash \widetilde{H}\right) \cup\left(E\left(\widetilde{R}_{j} \backslash \widetilde{H}\right)\right)\right.$ пусто, то есть каждая вершина графа $\widetilde{R}_{i} \backslash \widetilde{H}$ 
не соединена ни с одной вершиной графа $\widetilde{R}_{j} \backslash \tilde{H}$. Для этого мы сначала докажем, что существует такая константа $K_{1}$, что для каждого $\widetilde{R}_{i}$ в $\widetilde{G}$ существует не более чем $K_{1}$ точных $(G, H)$-расширений графа $\tilde{H}$, пересекающихся с $\widetilde{R}_{i}$ более чем по $\widetilde{H}$. После чего останется доказать, что в $\widetilde{G}$ число пар точных $(G, H)$-расширений графа $\tilde{H}$, между которыми есть ребро (множество $E\left(\widetilde{R}_{i} \cup \widetilde{R}_{j} \backslash \tilde{H}\right) \backslash\left(E\left(\widetilde{R}_{i} \backslash \tilde{H}\right) \cup\left(E\left(\widetilde{R}_{j} \backslash \tilde{H}\right)\right)\right.$ не пусто) ограничено некоторой константой $K_{2}$. Итак, оценим для некоторого графа $\widetilde{R}_{i}$ число $\eta_{1}\left(\widetilde{R}_{i}\right)$ таких графов $\widetilde{R}_{j}$, что $\widetilde{R}_{i} \cap \widetilde{R}_{j} \supset \widetilde{H}$. Пусть $\widetilde{R}_{j}-$ такой граф. Тогда для любого графа $X, \widetilde{R}_{i} \cap \widetilde{R}_{j} \subseteq X \subset \widetilde{R}_{i}$, выполнены соотношения $f(X, \tilde{H})>0$ и $f\left(\widetilde{R}_{i}, \tilde{H}\right)=0$. Поэтому в силу тождества (1) справедливо неравенство $f\left(\widetilde{R}_{i}, X\right)<0$ (если $\widetilde{R}_{i} \cap \widetilde{R}_{j}=\tilde{H}$ и $X=\widetilde{R}_{i} \cap \widetilde{R}_{j}$, то $\left.f\left(\widetilde{R}_{i}, X\right)=0\right)$. Следовательно, пара $\left(\widetilde{R}_{i}, \widetilde{R}_{i} \cap \widetilde{R}_{j}\right)$ является жесткой. Заметим, что число подграфов в $G$, содержащих $H$, ограничено константой $2^{l-k}$. С другой стороны, $\widetilde{G} \in \mathscr{S}_{1}$. Поэтому

$$
\eta_{1}\left(\widetilde{R}_{i}\right) \leqslant 2^{l-k} \max \{K(2(l-k), k+1), K(2(l-k), k+2), \ldots, K(2(l-k), l)\}=K_{1} .
$$

Оценим теперь число $\eta_{2}\left(\widetilde{R}_{i}, \widetilde{R}_{j}\right)$ таких пар графов $\left(\widetilde{R}_{i}, \widetilde{R}_{j}\right)$, которые пересекаются в точности по $\tilde{H}$, что между графами $\widetilde{R}_{i} \backslash \widetilde{H}$ и $\widetilde{R}_{j} \backslash \widetilde{H}$ есть ребро из множества $E(\widetilde{G})$. Итак, пусть $\left(\widetilde{R}_{i}, \widetilde{R}_{j}\right)$ - такая пара. Докажем, что пара $\left(\widetilde{R}_{i} \cup \widetilde{R}_{j}, \widetilde{H}\right)$ является $\alpha$-жесткой. Рассмотрим граф $X$, для которого $\widetilde{H} \subseteq X \subset \widetilde{R}_{i} \cup \widetilde{R}_{j}$. Положим $X_{i}=X \cap \widetilde{R}_{i}, X_{j}=X \cap \widetilde{R}_{j}$. Если $\widetilde{H} \subseteq X_{i} \subset \widetilde{R}_{i}, \widetilde{H} \subseteq X_{j} \subset \widetilde{R}_{j}$ (при этом только в одном из двух нестрогих включений достигается равенство), то справедливы неравенства $f\left(\widetilde{R}_{i}, X_{i}\right) \leqslant 0, f\left(\widetilde{R}_{j}, X_{j}\right) \leqslant 0$ (опять же, только в одном из этих неравенств достигается равенство). Поэтому $f\left(\widetilde{R}_{i} \cup \widetilde{R}_{j}, X\right)<0$. Если $X_{i}=\tilde{H}$ и $X_{j}=\tilde{H}$, то $X=\tilde{H}$, и в связи с наличием дополнительного ребра $f\left(\widetilde{R}_{i} \cup \widetilde{R}_{j}, X\right)<0$. Если, скажем, граф $X_{i}$ совпадает с графом $\widetilde{R}_{i}$, при этом граф $X_{j}$ отличен от $\tilde{H}$, то $f\left(\widetilde{R}_{i} \cup \widetilde{R}_{j}, X\right) \leqslant f\left(\widetilde{R}_{j}, X_{j}\right)<0$. Если же граф $X_{i}$ совпадает с графом $\widetilde{R}_{i}$, а граф $X_{j}$ совпадает с $\widetilde{H}$, то

$$
f\left(\widetilde{R}_{i} \cup \widetilde{R}_{j}, X\right) \leqslant f\left(\widetilde{R}_{j}, \widetilde{H}\right)-\alpha=-\alpha<0 .
$$

Таким образом, в каждом из случаев выполнено неравенство $f\left(\widetilde{R}_{i} \cup \widetilde{R}_{j}, X\right)<0$, то есть пара $\left(\widetilde{R}_{i} \cup \widetilde{R}_{j}, \widetilde{H}\right)$ является $\alpha$-жесткой. Вспомним теперь, что граф $\widetilde{G}$ содержится в множестве $\mathscr{Y}_{1}$. Следовательно, для любых вершин $\tilde{x}_{1}, \ldots, \tilde{x}_{k}$ графа $\widetilde{G}$ мощность множества $\left|V\left(c l_{2(l-k)}\left(\tilde{x}_{1}, \ldots, \tilde{x}_{k}\right)\right)\right|$ не превосходит $K(2(l-k), k)$. В силу сделанных выводов,

$$
\eta_{2}\left(\widetilde{R}_{i}, \widetilde{R}_{j}\right) \leqslant K(2(l-k), k)=K_{2} .
$$

Таким образом, мы можем утверждать, что в графе $\widetilde{G}$ найдется $\left(\begin{array}{c}m \\ \xi\end{array}\right) \geqslant \Theta\left(N^{\xi \beta / 2}\right)$ точных $(S, H)$-расширений графа $\widetilde{H}$. Граф $\widetilde{G}$ содержится в $\mathscr{S}_{2}$, поэтому число копий $S$ в нем не более, чем $\Theta\left(N^{k}\right)$. Но $\xi>2 k / \beta$. Получили противоречие, а следовательно, $\mathscr{Y}=\varnothing$.

Для завершения доказательства леммы осталось показать, что число $(G, H)$-расширений с дополнительным ребром ограничено константой. Но это так в силу леммы о жестких цепях. Действительно, если к нейтральной паре добавить ребро, то получится жесткая пара, а число соответствующих расширений ограничено константой. Лемма доказана.

Теперь мы можем обратиться непосредственно к доказательству того, что число с дополнительными ребрами бесконечно мало по сравнению с числом всех расширений. 


\subsection{2. Доказательство теоремы о точных надежных расширениях}

Требуется доказать, что если $(G, H)-\alpha$-надежная пара, то число всех $(G, H)$-расширений эквивалентно числу точных $(G, H)$-расширений.

Так как число различных способов добавить ребра в множество $E(G) \backslash E(H)$ является константой (не зависит от $N$ ), нам достаточно показать, что как бы мы ни добавляли ребра в это множество, число полученных расширений будет бесконечно мало по сравнению с числом точных расширений без добавленных ребер.

Итак, добавим в множество $E(G) \backslash E(H) \xi$ ребер. В результате граф $G$ заменится на граф $\widehat{G}$. Предположим, что в графе $\widehat{G} \backslash H$ найдется такой подграф $\widehat{S}$, что справедливо равенство $f(\widehat{G}, \widehat{G} \backslash \widehat{S})=0$, при этом множество ребер $E(\widehat{G}) \backslash(E(\widehat{G} \backslash \widehat{S}) \cup E(\widehat{S}))$ пусто. Положим $S=\left.G\right|_{V(\widehat{S})}, s=|V(S \cup H)|$. Тогда либо пара $(G, G \backslash S)$ является $\alpha$-надежной, либо $\rho(S)=1 / \alpha$. Во втором случае приходим к противоречию, так как если $f(S \cup H, H)=0$, то пара $(G, H)$ не является $\alpha$-надежной. В первом случае в силу леммы 3 и теоремы 4 найдется такое $\beta>0$, что с асимптотической вероятностью 1 для любых вершины $\tilde{x}_{1}, \ldots, \tilde{x}_{k}, \tilde{y}_{1}, \ldots, \tilde{y}_{s}$ справедливы соотношения

$$
\begin{aligned}
N_{(\widehat{G}, H)}\left(\tilde{x}_{1}, \ldots, \tilde{x}_{k}\right) & =N_{(\widehat{S} \cup H, H)}\left(\tilde{x}_{1}, \ldots, \tilde{x}_{k}\right) N_{(\widehat{G}, \hat{S} \cup H)}\left(\tilde{y}_{1}, \ldots, \tilde{y}_{s}\right) \\
& \leqslant N_{\widehat{S}} N_{(G, S \cup H)}\left(\tilde{x}_{1}, \ldots, \tilde{x}_{k}\right) \\
& =o\left(N^{\beta / 2} N^{-\beta} N_{(G, H)}\left(\tilde{x}_{1}, \ldots, \tilde{x}_{k}\right)\right) \\
& =o\left(N_{(G, H)}\left(\tilde{x}_{1}, \ldots, \tilde{x}_{k}\right)\right)
\end{aligned}
$$

Будем теперь считать, что для любого такого графа $\widehat{S}, \hat{S} \subseteq \widehat{G} \backslash H$, что множество ребер $E(\widehat{G}) \backslash(E(\widehat{G} \backslash \widehat{S}) \cup E(\widehat{S}))$ пусто, справедливо неравенство $f(\widehat{G}, \widehat{G} \backslash \widehat{S}) \neq 0$. Тогда в соответствии с леммой 2 нам нужно рассмотреть семь случаев:

(1) пара $(\widehat{G}, H)$ является $\alpha$-надежной;

(2) пара $(\widehat{G}, H)$ является $\alpha$-нейтральной цепью;

(3) пара $(\widehat{G}, H)$ является $\alpha$-жесткой;

(4) существует такой граф $\hat{X}, H \subset \widehat{X} \subset \widehat{G}$, что пара $(\hat{X}, H)$ является $\alpha$-нейтральной цепью, пара $(\widehat{G}, \hat{X})$ является $\alpha$-надежной;

(5) существует такой граф $\widehat{X}, H \subset \widehat{X} \subset \widehat{G}$, что пара $(\hat{X}, H)$ является $\alpha$-жесткой, пара $(\widehat{G}, \widehat{X})$ является $\alpha$-надежной;

(6) существует такой граф $\widehat{X}, H \subset \widehat{X} \subset \widehat{G}$, что пара $(\widehat{X}, H)$ является $\alpha$-жесткой, пара $(\widehat{G}, \hat{X})$ является $\alpha$-нейтральной цепью;

(7) существуют такие графы $\hat{X}, \hat{Y}, H \subset \widehat{Y} \subset \hat{X} \subset \widehat{G}$, что пара $(\hat{Y}, H)$ является $\alpha$-жесткой, пара $(\hat{X}, \hat{Y})$ является $\alpha$-нейтральной цепью, пара $(\widehat{G}, \widehat{X})$ является $\alpha$-надежной.

В первом случае, в силу того, что число ребер увеличилось, величина $f(G, H)$ уменьшилась на $\alpha \xi$, то есть $f(\widehat{G}, H)=f(G, H)-\alpha \xi$. Следовательно, по теореме 1 с асимптотической вероятностью 1 для любых вершин $\tilde{x}_{1}, \ldots, \tilde{x}_{k}$ справедливы равенства

$$
N_{(\widehat{G}, H)}\left(\tilde{x}_{1}, \ldots, \tilde{x}_{k}\right)=\Theta\left(N^{f(\widehat{G}, H)}\right)=o\left(N^{f(G, H)}\right)=o\left(N_{(G, H)}\left(\tilde{x}_{1}, \ldots, \tilde{x}_{k}\right)\right) .
$$


Во втором случае равенство

$$
N_{(\widehat{G}, H)}\left(\tilde{x}_{1}, \ldots, \tilde{x}_{k}\right)=o\left(N_{(G, H)}\left(\tilde{x}_{1}, \ldots, \tilde{x}_{k}\right)\right)
$$

справедливо в силу леммы о числе нейтральных расширений, в третьем случае - в силу леммы о жестких цепях.

Рассмотрим одновременно четвертый и пятый случай. Иными словами, либо пара $(\widehat{X}, H)$ является $\alpha$-нейтральной цепью, либо она $\alpha$-жесткая. Положим $|V(\hat{X})|=s$. Рассмотрим граф $X=\left.G\right|_{V(\widehat{X})}$. Тогда $\widehat{G}$ является $(G, X)$-расширением графа $\widehat{X}$, где пара $(G, X)$ является $\alpha$-надежной (так как пара $(\hat{G}, \hat{X})$ является $\alpha$-надежной, а множество $E(\widehat{G}) \backslash E(\widehat{X})$ содержит в себе множество $E(G) \backslash E(X))$. В силу леммы 3, найдется такое число $\beta>0$, что с асимптотической вероятностью 1 для любых вершин $\tilde{y}_{1}, \ldots, \tilde{y}_{s}$ и $\tilde{x}_{1}, \ldots, \tilde{x}_{k}$ справедливо равенство

$$
N_{(\widehat{G}, \widehat{X})}\left(\tilde{y}_{1}, \ldots, \tilde{y}_{s}\right)=O\left(N_{(G, H)}\left(\tilde{x}_{1}, \ldots, \tilde{x}_{k}\right) N^{-\beta}\right) .
$$

С другой стороны, в силу леммы 5 , в случае нейтральной цепи $(\hat{X}, H)$ (утверждение леммы 5, очевидно, выполнено и для нейтральных цепей) и леммы 4 в случае жесткой пары $(\hat{X}, H)$, с асимптотической вероятностью 1 для любых $\tilde{x}_{1}, \ldots, \tilde{x}_{k}$ справедливо равенство

$$
N_{(\hat{X}, H)}\left(\tilde{x}_{1}, \ldots, \tilde{x}_{k}\right)=o\left(N^{\beta / 2}\right) .
$$

Следовательно, с асимптотической вероятностью 1 для любых вершин $\tilde{x}_{1}, \ldots, \tilde{x}_{k}$ выполнены равенства

$$
N_{(\widehat{G}, H)}\left(\tilde{x}_{1}, \ldots, \tilde{x}_{k}\right)=o\left(N_{(G, H)}\left(\tilde{x}_{1}, \ldots, \tilde{x}_{k}\right) N^{-\beta / 2}\right)=o\left(N_{(G, H)}\left(\tilde{x}_{1}, \ldots, \tilde{x}_{k}\right)\right) .
$$

В шестом случае для любого положительного $\beta \leqslant f(G, H)$ с асимптотической вероятностью 1 для любых $\tilde{x}_{1}, \ldots, \tilde{x}_{k}$ справедлива цепочка равенств

$$
N_{(\widehat{G}, H)}\left(\tilde{x}_{1}, \ldots, \tilde{x}_{k}\right)=o\left(N^{\beta}\right)=o\left(N^{f(G, H)}\right)=o\left(N_{(G, H)}\left(\tilde{x}_{1}, \ldots, \tilde{x}_{k}\right)\right) .
$$

Пусть, наконец, существуют такие графы $\widehat{X}, \hat{Y}, H \subset \widehat{Y} \subset \widehat{X} \subset \widehat{G}$, что пара $(\widehat{Y}, H)$ является $\alpha$-жесткой, пара $(\hat{X}, \hat{Y})$ является $\alpha$-нейтральной цепью, пара $(\widehat{G}, \hat{X})$ является $\alpha$-надежной. Вспомним доказательство леммы 2. Если для каждого графа $\widehat{S}, H \subset \widehat{S} \subseteq \widehat{G}$, справедливо неравенство $f(\widehat{S}, H) \geqslant 0$, то задачу можно свести к предыдущим случаям. Если же существует граф $\widehat{S}, H \subset \widehat{S} \subseteq \widehat{G}$, для которого $f(\widehat{S}, H)<0$, то можно так подобрать подходящие под условие текущего случая графы $\hat{X}, \hat{Y}$, что для любого графа $\hat{R}, \hat{Y} \subset \widehat{R} \subseteq \widehat{G}$, справедливо неравенство $f(\hat{R}, \hat{Y}) \geqslant 0$. Пусть $\widehat{X}, \hat{Y}-$ такие графы. Положим $X=\left.G\right|_{V(\widehat{X})}, Y=\left.G\right|_{V(\widehat{Y})}$. Так как $E(G) \subset E(\widehat{G})$, неравенство $f(R, Y) \geqslant 0$ выполнено для каждого графа $R, Y \subset R \subseteq G$. Пусть $|V(Y)|=s$. Из четвертого случая следует, что с асимптотической вероятностью 1 для любых вершин $\tilde{y}_{1}, \ldots, \tilde{y}_{s}$ справедливо равенство

$$
N_{(\widehat{G}, \widehat{Y})}\left(\tilde{y}_{1}, \ldots, \tilde{y}_{s}\right)=o\left(N_{(G, Y)}\left(\tilde{y}_{1}, \ldots, \tilde{y}_{s}\right)\right) .
$$

Так как для любого графа $R, Y \subset R \subseteq G$, справедливо неравенство $f(R, Y) \geqslant 0$, вновь обращаясь к доказательству леммы 2, мы видим, что существуют такие графы $Z, W$, $Y \subseteq Z \subseteq W \subseteq G$, что пара $(Z, Y)$ является $\alpha$-нейтральной цепью, пара $(W, Z)$ является $\alpha$-надежной, $f(G, W)=0$, и $E(G) \backslash(E(G \backslash W) \cup E(W))=\varnothing$. Если $W \neq G$, то множество 
$E((G \backslash W) \cup H) \backslash(E(G \backslash W) \cup E(H))$ должно быть пустым, иначе $f((G \backslash W) \cup H, H)<0$ и пара $(G, H)$ не является $\alpha$-надежной. Но в этом случае $f((G \backslash W) \cup H, H)=0$ и пара $(G, H)$ снова не является $\alpha$-надежной. Поэтому графы $W$ и $G$ совпадают. Тогда, в силу леммы о числе нейтральных расширений и леммы о числе надежных расширений, существует такое $\beta>0$, что с асимптотической вероятностью 1 для любых вершин $\tilde{y}_{1}, \ldots, \tilde{y}_{s}, \tilde{x}_{1}, \ldots, \tilde{x}_{k}$ справедлива цепочка равенств

$$
\begin{aligned}
N_{(G, Y)}\left(\tilde{y}_{1}, \ldots, \tilde{y}_{s}\right) & =O\left(N_{(G, H)}\left(\tilde{x}_{1}, \ldots, \tilde{x}_{k}\right) N^{-\beta} N_{(Z, Y)}\left(\tilde{y}_{1}, \ldots, \tilde{y}_{s}\right)\right) \\
& =o\left(N_{(G, H)}\left(\tilde{x}_{1}, \ldots, \tilde{x}_{k}\right) N^{-\beta / 2}\right)=o\left(N_{(G, H)}\left(\tilde{x}_{1}, \ldots, \tilde{x}_{k}\right)\right) .
\end{aligned}
$$

В силу леммы 4 , существует такое число $K(s-k, k)$, что с асимптотической вероятностью 1 для любых вершин $\tilde{x}_{1}, \ldots, \tilde{x}_{k}$ выполнено неравенство

$$
N_{(\widehat{Y}, H)}\left(\tilde{x}_{1}, \ldots, \tilde{x}_{k}\right) \leqslant K(s-k, k) .
$$

Поэтому с асимптотической вероятностью 1 для любых вершин $\tilde{x}_{1}, \ldots, \tilde{x}_{k}$ выполнены равенства

$$
N_{(\widehat{G}, H)}\left(\tilde{x}_{1}, \ldots, \tilde{x}_{k}\right)=o\left(K(s-k, k) N_{(G, H)}\left(\tilde{x}_{1}, \ldots, \tilde{x}_{k}\right)\right)=o\left(N_{(G, H)}\left(\tilde{x}_{1}, \ldots, \tilde{x}_{k}\right)\right) .
$$

Теорема доказана.

Обратимся теперь непосредственно к доказательству теоремы 2. Используем индукцию, причем базой индукции будет служить предложение 1 , доказываемое в следующем параграфе.

\section{3. Доказательство предложения 1}

Рассмотрим графы $H, G, T, K$, для которых выполнены следующие свойства: $H \subset G$, $T \subset K,|V(T)| \leqslant|V(G)|$; пара $(G, H)$ является $\alpha$-надежной; пара $(K, T)$ является $\alpha$-нейтральной.

Рассмотрим, кроме того, графы $\widetilde{H}, \widetilde{G}, \widetilde{K}, \widetilde{T}$. Пусть $|V(\widetilde{H})|=|V(H)|=k, \widetilde{T} \subseteq \widetilde{G}$, $\widetilde{K} \cap \widetilde{G}=\widetilde{T}, \widetilde{H} \cap \widetilde{T} \neq \widetilde{T}$; $\widetilde{G}$ есть точное $(G, H)$-расширение графа $\widetilde{H}, \widetilde{K}$ есть точное $(K, T)$-расширение графа $\widetilde{T}$, множество $E((\widetilde{K} \cup \widetilde{G}) \backslash \widetilde{T}) \backslash(E(\widetilde{K} \backslash \widetilde{T}) \cup E(\widetilde{G} \backslash \widetilde{T}))$ пусто. Набор таких графов $(\widetilde{G}, \widetilde{H}, \widetilde{K}, \widetilde{T})$ будем называть $(G, H, K, T, 1)$-сетью.

Определим аналогичное понятие в случае, когда в построении участвует несколько $(K, T)$-расширений. Пусть $\left(\widetilde{G}, \widetilde{H}, \widetilde{K}_{1}, \widetilde{T}_{1}\right), \ldots,\left(\widetilde{G}, \widetilde{H}, \widetilde{K}_{r}, \widetilde{T}_{r}\right)$ являются $(G, H, K, T, 1)$ сетями. Пусть, кроме того, между любыми двумя графами из множества $\left\{\widetilde{K}_{i} \backslash \widetilde{T}_{i}\right\}_{i \in\{1, \ldots, r\}}$ нет ребер, и для всех $i \neq j$ множества $V\left(\widetilde{K}_{i} \backslash \widetilde{T}_{i}\right)$ и $V\left(\widetilde{K}_{j} \backslash \widetilde{T}_{j}\right)$ не пересекаются. Тогда набор $\left(\widetilde{G}, \widetilde{H}, \widetilde{K}_{i}, \widetilde{T}_{i}, i \in\{1, \ldots, r\}\right)$ будем называть $(G, H, K, T, r)$-сетью.

Рассмотрим графы $\Gamma, \widetilde{G}, \Gamma \supset \widetilde{G}$. Пусть $\widetilde{G}-$ точное $(G, H)$-расширение графа $\widetilde{H}$. Рассмотрим все $(G, H, K, T, 1)$-сети вида $\left(\widetilde{G}, \widetilde{H}, \widetilde{K}_{i}, \widetilde{T}_{i}\right), i \in\{1, \ldots, M\}$, где все графы $\widetilde{K}_{i}, \widetilde{T}_{i}$ содержатся в $\Gamma$. Определим максимальный отделимый подграф $U(\Gamma, \widetilde{G}, \widetilde{H}, K, T)$. Пусть $\mathscr{F} \subset 2^{\{1, \ldots, M\}}$ - такое множество подмножеств в $\{1, \ldots, M\}$, что для любого $I \in \mathscr{g}$ и любых $i, j \in I, i \neq j$, граф $\left(\widetilde{G} \cup \widetilde{K}_{i}\right) \cap\left(\widetilde{G} \cup \widetilde{K}_{j}\right)$ совпадает с графом $\widetilde{G}$ и множество ребер $E\left(\left(\widetilde{K}_{i} \backslash \widetilde{T}_{i}\right) \cup\left(\widetilde{K}_{j} \backslash \widetilde{T}_{j}\right)\right) \backslash\left(E\left(\widetilde{K}_{i} \backslash \widetilde{T}_{i}\right) \cup E\left(\widetilde{K}_{j} \backslash \widetilde{T}_{j}\right)\right)$ пусто. Пусть $\hat{I} \in \mathscr{g}-$ любое множество, для которого $|\hat{I}|=\max \{|I|: I \in \mathscr{F}\}$. Тогда

$$
U(\Gamma, \widetilde{G}, \widetilde{H}, K, T)=\left(\bigcup_{i \in \hat{I}} \widetilde{K}_{i}\right) \cup \widetilde{G} .
$$


Если пара графов $\widetilde{K}_{1}, \widetilde{K}_{2}$ обладает свойствами пар графов, принадлежащих максимальному отделимому подграфу, то будем говорить, что эта пара обладает свойством отделимости (граф $\left(\widetilde{G} \cup \widetilde{K}_{1}\right) \cap\left(\widetilde{G} \cup \widetilde{K}_{2}\right)$ совпадает с графом $\widetilde{G}$ и множество ребер $E\left(\left(\widetilde{K}_{1} \backslash \widetilde{T}_{1}\right) \cup\left(\widetilde{K}_{2} \backslash \widetilde{T}_{2}\right)\right) \backslash\left(E\left(\widetilde{K}_{1} \backslash \widetilde{T}_{1}\right) \cup E\left(\widetilde{K}_{2} \backslash \widetilde{T}_{2}\right)\right)$ пусто); про какие графы $\widetilde{G}, \widetilde{T}_{1}$, $\widetilde{T}_{2}$ идет речь, при этом будет ясно из контекста.

Обозначим через $d_{r}(\Gamma, \widetilde{G}, \widetilde{H}, K, T)$ есть число различных $(G, H, K, T, r)$-сетей вида $\left(\widetilde{G}, \widetilde{H}, \widetilde{K}_{i}, \widetilde{T}_{i}, i \in\{1, \ldots, r\}\right)$, где все графы $\widetilde{K}_{i}$ есть подграфы графа $U(\Gamma, \widetilde{G}, \widetilde{H}, K, T)$. Если в графе $U(\Gamma, \widetilde{G}, \widetilde{H}, K, T)$ не содержится таких подграфов, образующих $(G, H, K, T, r)$ сеть описанного вида, то будем считать, что $d_{r}(\Gamma, \widetilde{G}, \widetilde{H}, K, T)=0$. Конечно, максимальный отделимый подграф определяется неоднозначно, но однозначно определяется число вершин в нем и число образующих его графов $\widetilde{K}_{i}$ (это число равно $|\hat{I}|$ ). Из этого следует, что величина $d_{r}(\Gamma, \widetilde{G}, \widetilde{H}, K, T)$ определена однозначно. Следующее рассуждение окончательно прояснит этот факт.

Пусть $d_{1}(\Gamma, \widetilde{G}, \widetilde{H}, K, T) \neq 0$, тогда

$$
|\hat{I}|=\max \left\{r: d_{r}(\Gamma, \widetilde{G}, \widetilde{H}, K, T) \neq 0\right\},
$$

то есть для каждого $r>|\hat{I}|$ справедливо равенство $d_{r}(\Gamma, \widetilde{G}, \widetilde{H}, K, T)=0$. Тогда, очевидно, для каждого $r \in\{1, \ldots,|\hat{I}|\}$ выполнено равенство $d_{r}(\Gamma, \widetilde{G}, \widetilde{H}, K, T)=\left(\begin{array}{c}|\hat{I}| \\ r\end{array}\right)$ (из этого следует, что величина $d_{r}(\Gamma, \widetilde{G}, \widetilde{H}, K, T)$ определена однозначно). Поэтому, если $d_{1}(\Gamma, \widetilde{G}, \widetilde{H}, K, T) \neq 0$, то справедливо тождество

$$
\sum_{r \geqslant 1}(-1)^{r-1} d_{r}(\Gamma, \widetilde{G}, \widetilde{H}, K, T)=1 .
$$

Пусть $D_{\widetilde{H}}(\Gamma, G, H, K, T)$ - число точных $(G, H)$-расширений $\widetilde{G}$ графа $\widetilde{H}$ в графе $\Gamma$, для которых число $d_{1}(\Gamma, \widetilde{G}, \widetilde{H}, K, T)$ отлично от нуля. В силу сделанных выводов несложно видеть, что выполнено равенство

$$
D_{\widetilde{H}}(\Gamma, G, H, K, T)=\sum_{r \geqslant 1} \sum_{\widetilde{G}}(-1)^{r-1} d_{r}(\Gamma, \widetilde{G}, \widetilde{H}, K, T),
$$

где суммирование ведется по всем таким графам $\widetilde{G}$, являющимся точными $(G, H)$-расширениями графа $\widetilde{H}$, что $\widetilde{H} \subset \widetilde{G} \subset \Gamma$. Таким образом, доказано следующее утверждение.

Предложение 2. Для таких графов $Г, \widetilde{H}$, что $\widetilde{H} \subset \Gamma,|V(\widetilde{H})|=|V(H)|$, справедливо тождество (2).

Рассмотрим случайный граф $G(N, p)$, где $p=N^{-\alpha}, 0<\alpha \leqslant 1$, и $\alpha$ есть рациональное число. Пусть, как и раньше,

$$
|V(G)|=l, \quad|V(H)|=k .
$$

Положим также

$$
|V(K \backslash T)|=t, \quad|V(T)|=m .
$$

Обозначим через $N_{(G, H, K, T, r)}\left(\tilde{x}_{1}, \ldots, \tilde{x}_{k}\right)$ сумму по всем $\widetilde{G}$ в случайном графе, являющимися точными $(G, H)$-расширениями подграфа, индуцированного на $\left\{\tilde{x}_{1}, \ldots, \tilde{x}_{k}\right\}$, чисел $(G, H, K, T, r)$-сетей вида $\left(\widetilde{G},\left.\mathscr{G}\right|_{\left\{\tilde{x}_{1}, \ldots, \tilde{x}_{k}\right\}}, \widetilde{K}_{i}, \widetilde{T}_{i}, i \in\{1, \ldots, r\}\right)$. Иными словами, пусть 
$W_{G}, W_{T_{1}}, \ldots, W_{T_{r}}, W_{K_{1}}, \ldots, W_{K_{r}} \subset \mathscr{V}-$ множества, обладающие следующими свойствами:

$$
\begin{gathered}
W_{G} \cap\left\{\tilde{x}_{1}, \ldots, \tilde{x}_{k}\right\}=\varnothing, \quad W_{T_{i}} \cap\left\{\tilde{x}_{1}, \ldots, \tilde{x}_{k}\right\} \neq W_{T_{i}}, \\
W_{T_{i}} \subseteq W_{G} \cup\left\{\tilde{x}_{1}, \ldots, \tilde{x}_{k}\right\}, \quad W_{K_{i}} \cap\left(W_{G} \cup\left\{\tilde{x}_{1}, \ldots, \tilde{x}_{k}\right\}\right)=\varnothing, \\
W_{K_{i}} \cap W_{K_{j}}=\varnothing, \quad\left|W_{G}\right|=l-k, \quad\left|W_{T_{i}}\right|=m, \quad\left|W_{K_{i}}\right|=t .
\end{gathered}
$$

Пусть $\mathbf{W}-$ множество всех наборов из $2 r+1$ множеств, удовлетворяющих перечисленным свойствам. Если граф $\left.\mathscr{G}\right|_{\left\{\tilde{x}_{1}, \ldots, \tilde{x}_{k}\right\} \cup W_{G}}$ является точным $(G, H)$-расширением графа $\left.\mathscr{G}\right|_{\left\{\tilde{x}_{1}, \ldots, \tilde{x}_{k}\right\}}$, графы $\left.\right|_{W_{K_{i}} \cup W_{T_{i}}}$ являются точными $(K, T)$-расширениями графов $G_{W_{T_{i}}}$, и

$$
\begin{aligned}
& E\left(\left.\left.\mathscr{G}\right|_{W_{K_{i}}} \cup \mathscr{G}\right|_{W_{K_{j}}}\right) \backslash\left(E\left(\left.\mathscr{G}\right|_{W_{K_{i}}}\right) \cup E\left(\left.\mathscr{G}\right|_{W_{K_{j}}}\right)\right)=\varnothing,
\end{aligned}
$$

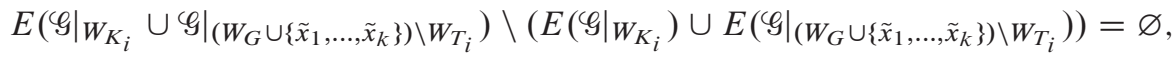

то положим

$$
I_{W_{G}, W_{T_{i}}, W_{K_{i}}, i \in\{1, \ldots, r\}}(\mathscr{G})=1 .
$$

В противном случае положим

$$
I_{W_{G}, W_{T_{i}}, W_{K_{i}}, i \in\{1, \ldots, r\}}(\mathscr{G})=0 .
$$

Тогда случайная величина $N_{(G, H, K, T, r)}\left(\tilde{x}_{1}, \ldots, \tilde{x}_{k}\right)$ определяется равенством

$$
N_{(G, H, K, T, r)}\left(\tilde{x}_{1}, \ldots, \tilde{x}_{k}\right)=\sum_{\left(W_{G}, W_{T_{1}}, \ldots, W_{T_{r}}, W_{K_{1}}, \ldots, W_{K_{r}}\right) \in \mathbf{W}} I_{W_{G}, W_{T_{i}}, W_{K_{i}}, i \in\{1, \ldots, r\}} .
$$

Предложение 3. Для любого $\varepsilon>0$ найдется такое число $r_{0} \in \mathbf{N}$, что для любых $r>r_{0}$ с асимптотической вероятностью 1 для любых вершин $\tilde{x}_{1}, \ldots, \tilde{x}_{k}$ справедливы соотношения

$$
\begin{aligned}
N_{(G, H, K, T, r)}\left(\tilde{x}_{1}, \ldots, \tilde{x}_{k}\right) & \sim \mathbf{E}_{N, p} N_{(G, H, K, T, r)}\left(\tilde{x}_{1}, \ldots, \tilde{x}_{k}\right) \\
& =\Theta\left(N^{f(G, H)}\right) \leqslant \varepsilon N^{f(G, H)} .
\end{aligned}
$$

Доказательство. Пусть $S_{r}^{1}(G, H, K, T), \ldots, S_{r}^{j}(G, H, K, T)$ есть всевозможные различные $(G, H, K, T, r)$-сети вида $\left(G, H, K_{i}, T_{i}, i \in\{1, \ldots, r\}\right)$. Пусть $g\left(S_{r}^{h}(G, H, K, T)\right)$, где $S_{r}^{h}(G, H, K, T)=\left(G, H, K_{i}^{h}, T_{i}^{h}, i \in\{1, \ldots, r\}\right)$, есть число всевозможных точных $(G, H)$-расширений $G_{0}$ в $G \cup K_{1}^{h} \cup \ldots \cup K_{r}^{h}$ графа $H$, для которых существуют такие графы $K_{0, i}^{h}, T_{0, i}^{h}, i \in\{1, \ldots, r\}$, что набор $\left(G_{0}, H, K_{0, i}^{h}, T_{0, i}^{h}, i \in\{1, \ldots, r\}\right)$ есть $(G, H, K, T, r)$ сеть и справедливо равенство

$$
G_{0} \cup K_{0,1}^{h} \cup \ldots \cup K_{0, r}^{h}=G \cup K_{1}^{h} \cup \ldots \cup K_{r}^{h} .
$$

Для каждой сети $S_{r}^{h}(G, H, K, T)=\left(G, H, K_{i}^{h}, T_{i}^{h}, i \in\{1, \ldots, r\}\right)$ положим

$$
X_{r}^{h}(G, H, K, T)=G \cup K_{1}^{h} \cup \ldots \cup K_{r}^{h} .
$$

В силу леммы 1 , для любой $(G, H, K, T, r)$-сети вида $\left(G, H, K_{i}^{h}, T_{i}^{h}, i \in\{1, \ldots, r\}\right)$ пара $\left(G \cup K_{1}^{h} \cup \ldots \cup K_{r}^{h}, H\right)$ является $\alpha$-надежной. При этом справедливо равенство

$$
f\left(G \cup K_{1}^{h} \cup \ldots \cup K_{r}^{h}, H\right)=f\left(X_{r}^{h}(G, H, K, T), H\right)=f(G, H) .
$$


С одной стороны, в силу теоремы 3 и теоремы 1 , для любой $\alpha$-надежной пары $(X, H)$ с асимптотической вероятностью 1 для любых вершин $\tilde{x}_{1}, \ldots, \tilde{x}_{k}$ выполнено соотношение

$$
N_{(X, H)}^{\text {strict }}\left(\tilde{x}_{1}, \ldots, \tilde{x}_{k}\right) \sim \mathbf{E}_{N, p}\left(N_{(X, H)}\left(\tilde{x}_{1}, \ldots, \tilde{x}_{k}\right)\right) .
$$

С другой стороны, для любой $r$-сети $S_{r}^{h}(G, H, K, T)$ справедливо неравенство

$$
g\left(S_{r}^{h}(G, H, K, T)\right) \leqslant\left(\begin{array}{c}
r t+(l-k) \\
l-k
\end{array}\right)(l-k) ! .
$$

Поэтому

$$
\begin{aligned}
& N_{(G, H, K, T, r)}\left(\tilde{x}_{1}, \ldots, \tilde{x}_{k}\right) \\
& \quad=\sum_{h=1}^{j} N_{\left(X_{r}^{h}(G, H, K, T), H\right)}^{\text {strict }}\left(\tilde{x}_{1}, \ldots, \tilde{x}_{k}\right) g\left(S_{r}^{h}(G, H, K, T)\right) \\
& \quad \sim \sum_{h=1}^{j} \mathbf{E}_{N, p}\left(N_{\left(X_{r}^{h}(G, H, K, T), H\right)}\left(\tilde{x}_{1}, \ldots, \tilde{x}_{k}\right)\right) g\left(S_{r}^{h}(G, H, K, T)\right) \\
& \leqslant\left(\begin{array}{c}
r t+(l-k) \\
l-k
\end{array}\right)(l-k) ! \sum_{h=1}^{j} \mathbf{E}_{N, p}\left(N_{\left(X_{r}^{h}(G, H, K, T), H\right)}\left(\tilde{x}_{1}, \ldots, \tilde{x}_{k}\right)\right) .
\end{aligned}
$$

Из этой цепочки соотношений, в частности, следует, что

$$
\begin{aligned}
N_{(G, H, K, T, r)}\left(\tilde{x}_{1}, \ldots, \tilde{x}_{k}\right) & \sim \mathbf{E}_{N, p} N_{(G, H, K, T, r)}\left(\tilde{x}_{1}, \ldots, \tilde{x}_{k}\right) \\
& =\Theta\left(N^{f(G, H)}\right) .
\end{aligned}
$$

Для любых $\tilde{x}_{1}, \ldots, \tilde{x}_{k}$ выполнено неравенство

$$
\begin{aligned}
\sum_{h=1}^{j} \mathbf{E}_{N, p}\left(N _ { ( X _ { r } ^ { h } ( G , H , K , T ) , H ) } \left(\tilde{x}_{1}, \ldots,\right.\right. & \left.\left.\tilde{x}_{k}\right)\right) \\
& \leqslant \frac{(l-k) !(t !)^{r}}{(l-k+t r) !}\left(\begin{array}{c}
l-k+t r \\
l-k
\end{array}\right) \frac{(t r) !}{r !(t !)^{r}}\left(\begin{array}{c}
l \\
m
\end{array}\right)^{r} N^{f(G, H) .}
\end{aligned}
$$

Следовательно, при достаточно больших $r$ для любых $\tilde{x}_{1}, \ldots, \tilde{x}_{k}$ справедливы соотношения

$$
\begin{aligned}
& \sum_{h=1}^{j} \mathbf{E}_{N, p}\left(N_{\left(X_{r}^{h}(G, H, K, T), H\right)}\left(\tilde{x}_{1}, \ldots, \tilde{x}_{k}\right)\right) g\left(S_{r}^{h}(G, H, K, T)\right) \\
& \leqslant \frac{(l-k+t r) !}{r !(t r) !}\left(\begin{array}{c}
l \\
m
\end{array}\right)^{r} N^{f(G, H)} \leqslant \frac{1}{z !} 2^{l-k}(t r)^{l-k}\left(\begin{array}{c}
l \\
m
\end{array}\right)^{r} N^{f(G, H)}
\end{aligned}
$$

Поэтому для любого $\varepsilon>0$ найдется такое $r_{0} \in \mathbf{N}$, что для любых $r>r_{0}$ с асимптотической вероятностью 1 для любых вершин $\tilde{x}_{1}, \ldots, \tilde{x}_{k}$ справедливо неравенство

$$
N_{(G, H, K, T, r)}\left(\tilde{x}_{1}, \ldots, \tilde{x}_{k}\right) \leqslant \varepsilon N^{f(G, H)} .
$$

Утверждение доказано. 
С помощью этого утверждения мы сможем оценить число $(G, H)$-расширений, не являющихся максимальными. Для этого определим новые случайные величины. Для каждого набора вершин $\tilde{x}_{1}, \ldots, \tilde{x}_{k} \in \mathscr{V}$ обозначим $D_{(G, H, K, T)}\left(\tilde{x}_{1}, \ldots, \tilde{x}_{k}\right)$ случайную величину,

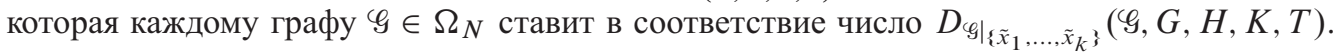
Случайная величина $d_{(G, H, K, T, r)}\left(\tilde{x}_{1}, \ldots, \tilde{x}_{k}\right)$ каждому графу $\mathscr{G} \in \Omega_{N}$ ставит в соответствие число $\sum_{\widetilde{G}} d_{r}(\mathscr{G}, \widetilde{G}, \widetilde{H}, K, T)$, где сумма берется по всем $\widetilde{G},\left.\mathscr{G}\right|_{\left\{\tilde{x}_{1}, \ldots, \tilde{x}_{k}\right\}} \subset \widetilde{G} \subset \mathscr{G}$, являющимся точными $(G, H)$-расширениями графа $\left.G_{\{} \tilde{x}_{1}, \ldots, \tilde{x}_{k}\right\}$.

Покажем, что для любого $r>1$ и любых вершин $\tilde{x}_{1}, \ldots, \tilde{x}_{k}$ справедливы неравенства

$$
\begin{aligned}
\sum_{i=1}^{2 r-2}(-1)^{i-1} d_{(G, H, K, T, i)}\left(\tilde{x}_{1}, \ldots, \tilde{x}_{k}\right) & \leqslant D_{(G, H, K, T)}\left(\tilde{x}_{1}, \ldots, \tilde{x}_{k}\right) \\
& \leqslant \sum_{i=1}^{2 r-1}(-1)^{i-1} d_{(G, H, K, T, i)}\left(\tilde{x}_{1}, \ldots, \tilde{x}_{k}\right) .
\end{aligned}
$$

Неравенство здесь понимается в следующем смысле: случайная величина $X_{1}$ на вероятностном пространстве $\Omega$ больше величины $X_{2}$, если для каждого $\omega \in \Omega$ выполнено неравенство $X_{1}(\omega)>X_{2}(\omega)$.

Пусть $\mathscr{G} \in \Omega_{N}, \widetilde{G}-$ точное $(G, H)$-расширение в графе $\mathscr{G}$ графа $\tilde{H}=\left.\mathscr{G}\right|_{\left\{\tilde{x}_{1}, \ldots, \tilde{x}_{k}\right\}}$. Положим

$$
\xi(\widetilde{G})=\frac{1}{t}(|V(U(\varphi, \widetilde{G}, \tilde{H}, K, T))|-l) .
$$

При $\xi(\widetilde{G}) \geqslant 1$ и $r \leqslant(\xi(\widetilde{G})+1) / 2$ справедливы неравенства

$$
\sum_{i=1}^{2 r-2}(-1)^{i-1}\left(\begin{array}{c}
\xi(\tilde{G}) \\
i
\end{array}\right) \leqslant \sum_{i=1}^{\xi(\widetilde{G})}(-1)^{i-1}\left(\begin{array}{c}
\xi(\tilde{G}) \\
i
\end{array}\right) \leqslant \sum_{i=1}^{2 r-1}(-1)^{i-1}\left(\begin{array}{c}
\xi(\tilde{G}) \\
i
\end{array}\right)
$$

Так как

$$
\left(\begin{array}{c}
\xi(\widetilde{G}) \\
i
\end{array}\right)=d_{i}(\varphi, \widetilde{G}, \tilde{H}, K, T),
$$

в силу предложения 1 для любого $r>1$ выполнены неравенства

$$
\begin{aligned}
\sum_{\widetilde{G}} \sum_{i=1}^{2 r-2}(-1)^{i-1} d_{i}(\mathscr{G}, \widetilde{G}, \tilde{H}, K, T) & \leqslant D_{\widetilde{H}}(\mathscr{G}, G, H, K, T) \\
& \leqslant \sum_{\widetilde{G}} \sum_{i=1}^{2 r-1}(-1)^{i-1} d_{i}(\mathscr{G}, \widetilde{G}, \tilde{H}, K, T) .
\end{aligned}
$$

Суммирование в обоих случаях ведется по графам $\widetilde{G}, \tilde{H} \subset \widetilde{G} \subset \mathscr{G}$, являющимся точными $(G, H)$-расширениями графа $\widetilde{H}$. Это объясняет справедливость неравенств (3).

Возьмем математическое ожидание всех случайных величин в этом неравенстве:

$$
\begin{aligned}
\sum_{i=1}^{2 r-2}(-1)^{i-1} \mathbf{E}_{N, p} d_{(G, H, K, T, i)}\left(\tilde{x}_{1}, \ldots, \tilde{x}_{k}\right) & \leqslant \mathbf{E}_{N, p} D_{(G, H, K, T)}\left(\tilde{x}_{1}, \ldots, \tilde{x}_{k}\right) \\
& \leqslant \sum_{i=1}^{2 r-1}(-1)^{i-1} \mathbf{E}_{N, p} d_{(G, H, K, T, i)}\left(\tilde{x}_{1}, \ldots, \tilde{x}_{k}\right) .
\end{aligned}
$$


Если бы в неравенствах (3), (4) вместо случайных величин $d_{(G, H, K, T, r)}\left(\tilde{x}_{1}, \ldots, \tilde{x}_{k}\right)$ стояли $N_{(G, H, K, T, r)}\left(\tilde{x}_{1}, \ldots, \tilde{x}_{k}\right)$, то можно было бы рассмотреть разность $D_{(G, H, K, T)}\left(\tilde{x}_{1}, \ldots, \tilde{x}_{k}\right)-$ $\mathbf{E}_{N, p} D_{(G, H, K, T)}\left(\tilde{x}_{1}, \ldots, \tilde{x}_{k}\right)$ и воспользоваться предложением 2 для ее оценки. Поэтому требуется установить, что случайная величина $d_{(G, H, K, T, r)}\left(\tilde{x}_{1}, \ldots, \tilde{x}_{k}\right)$ эквивалентна своему математическому ожиданию для любых вершин $\tilde{x}_{1}, \ldots, \tilde{x}_{k}$. Легко заметить, что для любых вершин $\tilde{x}_{1}, \ldots, \tilde{x}_{k}$ справедливо неравенство

$$
d_{(G, H, K, T, r)}\left(\tilde{x}_{1}, \ldots, \tilde{x}_{k}\right) \leqslant N_{(G, H, K, T, r)}\left(\tilde{x}_{1}, \ldots, \tilde{x}_{k}\right) .
$$

Поэтому, в силу предложения 2, достаточно сформулировать и обосновать следующий факт.

Предложение 4. Для любого $r \in \mathbf{N}$ с асимптотической вероятностью 1 для любых вершин $\tilde{x}_{1}, \ldots, \tilde{x}_{k}$ справедливо соотношение

$$
N_{(G, H, K, T, r)}\left(\tilde{x}_{1}, \ldots, \tilde{x}_{k}\right) \sim d_{(G, H, K, T, r)}\left(\tilde{x}_{1}, \ldots, \tilde{x}_{k}\right) .
$$

Доказательство. Заметим сперва, что пары точных $(K, T)$-расширений, не обладающие свойством отделимости, формируют жесткую пару. А именно, пусть $T_{i} \subset G, T_{i} \cap H \neq T_{i}$, $i \in\{1,2\}$. Пусть, кроме того, $K_{i}$ - точные $(K, T)$-расширения графов $T_{i}$, и множества $E\left(\left(K_{i} \cup G\right) \backslash T_{i}\right) \backslash\left(E\left(K_{i} \backslash T_{i}\right) \cup E\left(G \backslash T_{i}\right)\right)$ являются пустыми. Тогда, если пересечение графов $K_{1} \backslash T_{1}$ и $K_{2} \backslash T_{2}$ или множество $E\left(\left(K_{1} \backslash T_{1}\right) \cup\left(K_{2} \backslash T_{2}\right)\right) \backslash\left(E\left(K_{1} \backslash T_{1}\right) \cup E\left(K_{2} \backslash T_{2}\right)\right)$ непусто, то пара $\left(K_{1} \cup K_{2}, T_{1} \cup T_{2}\right)$ является $\alpha$-жесткой. Этот факт обоснован при доказательстве леммы 5.

Пусть $S_{r}^{h}(G, H, K, T), k \in\{1, \ldots, j\},-$ сети из доказательства предложения 2. Положим $g=\max \left\{g\left(S_{r}^{h}(G, H, K, T)\right), h \in\{1, \ldots, j\}\right\}$. В соответствии с тем же предложением 2, для любых вершин $\tilde{x}_{1}, \ldots, \tilde{x}_{k} \in \mathscr{V}$ выполнено равенство

$$
\mathbf{E}_{N, p} N_{(G, H, K, T, r)}\left(\tilde{x}_{1}, \ldots, \tilde{x}_{k}\right)=\Theta\left(N^{f(G, H)}\right) .
$$

Поэтому существует такая константа $c$, что

$$
\mathbf{E}_{N, p} N_{(G, H, K, T, r)}\left(\tilde{x}_{1}, \ldots, \tilde{x}_{k}\right) \geqslant c N^{f(G, H)} .
$$

Рассмотрим произвольное $\varepsilon_{0}>0$. В силу предложения 2 , найдется такое $N_{0}$, что для любого $N>N_{0}$ в $\Omega_{N}$ существует такое подмножество $\mathscr{S}_{0}(N)$, что

$$
\lim _{N \rightarrow \infty} \mathbf{P}_{N, p}\left(\mathscr{S}_{0}(N)\right)=1
$$

и для любого графа $\mathscr{G} \in \mathscr{Y}_{0}(N)$ и любых вершин $\tilde{x}_{1}, \ldots, \tilde{x}_{k} \in \mathscr{V}$ справедливы неравенства

$$
\begin{aligned}
\left(1-\varepsilon_{0}\right) N_{(G, H, K, T, r)}\left(\tilde{x}_{1}, \ldots, \tilde{x}_{k}\right) & \leqslant \mathbf{E}_{N, p} N_{(G, H, K, T, r)}\left(\tilde{x}_{1}, \ldots, \tilde{x}_{k}\right) \\
& \leqslant\left(1+\varepsilon_{0}\right) N_{(G, H, K, T, r)}\left(\tilde{x}_{1}, \ldots, \tilde{x}_{k}\right) .
\end{aligned}
$$

Пусть $\left\{\left(Y_{i}, H\right), i \in\{1, \ldots, \mu\}\right\}-$ множество, состоящее из всех возможных $\alpha$-надежных пар, у которых $\left|V\left(Y_{i}\right)\right| \leqslant r t+l, f\left(Y_{i}, H\right)=f(G, H)$ для всех $i$. Определим, кроме того, для каждого $i$ множество $\left\{\left(X_{j}^{i}, T_{j}^{i}\right), j \in\{1, \ldots, v\}\right\} \alpha$-жестких пар, для которых

$$
\left|V\left(X_{j}^{i}\right) \backslash V\left(T_{j}^{i}\right)\right| \leqslant 2 t, \quad\left|V\left(T_{j}^{i}\right)\right| \leqslant 2 m, \quad X_{j}^{i} \cap Y_{i}=T_{j}^{i} .
$$

В [2] (см. лемму 10.7.6) доказано, что для любого $\varepsilon_{1}>0$ найдется такое $N_{1}\left(\varepsilon_{1}\right)$, что для всех $N>N_{1}\left(\varepsilon_{1}\right)$ существует множество $\mathscr{S}_{1}(N) \subset \Omega_{N}$ такое, что

$$
\lim _{N \rightarrow \infty} \mathbf{P}_{N, p}\left(\mathscr{Y}_{1}(N)\right)=1,
$$


и обладающее следующим свойством: для каждого графа $\mathscr{G} \in \mathscr{Y}_{1}(N)$ и для любых вершин $\tilde{x}_{1}, \ldots, \tilde{x}_{k} \in \mathscr{V}$ суммарное по $i \in\{1, \ldots, \mu\}$ и $j \in\{1, \ldots, v\}$ число точных $\left(Y_{i} \cup X_{j}^{i}, H\right)$ расширений графа $\left.\mathscr{G}\right|_{\left\{\tilde{x}_{1}, \ldots, \tilde{x}_{k}\right\}}$ в $\mathscr{G}$ ограничено величиной $\varepsilon_{1} N^{f(G, H)}$. Положим

$$
\varepsilon_{1}=\frac{\varepsilon_{0} c}{g\left(1+\varepsilon_{0}\right)}
$$

Пусть $N>\max \left\{N_{0}, N_{1}\left(\varepsilon_{1}\right)\right\}$ и $\mathscr{G} \in \mathscr{I}_{0}(N) \cap \mathscr{I}_{1}(N), \tilde{x}_{1}, \ldots, \tilde{x}_{k} \in \mathscr{V}$. Пусть, кроме того, $X_{r}^{h}(G, H, K, T)$ - графы из предложения 2. Для того, чтобы для некоторого точного $(G, H)$-расширения $\widetilde{G}$ графа $\tilde{H}=G_{\left\{\tilde{x}_{1}, \ldots, \tilde{x}_{k}\right\}}$ точное $\left(X_{r}^{h}(G, H, K, T), G\right)$-расширение $\widetilde{X}_{r}^{h}$ графа $\widetilde{G}$ не содержалось в $U(\mathscr{G}, \widetilde{G}, \widetilde{H}, K, T)$, необходимо, чтобы существовали графы $\widetilde{K}_{0}, \widetilde{K}_{1}$, являющиеся точными $(K, T)$-расширениями графов $\widetilde{T}_{0}, \widetilde{T}_{1}$ в $\mathscr{G}$, не обладающие свойством отделимости, где графы $\widetilde{K}_{1}, \widetilde{T}_{0}, \widetilde{T}_{1}$ являются подграфами графа $\widetilde{X}_{r}^{h}$, граф $\widetilde{K}_{0} \backslash \widetilde{T}_{0}$ не пересекается с графом $\widetilde{X}_{r}^{h}$. Тогда пара $\left(\widetilde{K}_{0} \cup \widetilde{K}_{1}, \widetilde{T}_{0} \cup \widetilde{T}_{1}\right)$ является $\alpha$-жесткой, а пара $\left(\tilde{X}_{r}^{h} \backslash\left(\tilde{K}_{1} \backslash \widetilde{T}_{1}\right), \tilde{H}\right)-\alpha$-надежной, причем

$$
f\left(\tilde{X}_{r}^{h} \backslash\left(\tilde{K}_{1} \backslash \tilde{T}_{1}\right), \tilde{H}\right)=f(G, H) .
$$

Поэтому сумма по всем $h$ и всем точным $(G, H)$-расширениям $\widetilde{G}$ графа $\widetilde{H}$ чисел точных $\left(X_{r}^{h}(G, H, K, T), G\right)$-расширений графа $\widetilde{G}$, не содержащихся в $U(\mathscr{G}, \widetilde{G}, \widetilde{H}, K, T)$, ограничена величиной $g \varepsilon_{1} N^{f(G, H)}$. Следовательно,

$$
\begin{aligned}
N_{(G, H, K, T, r)} & \left(\tilde{x}_{1}, \ldots, \tilde{x}_{k}\right)-d_{(G, H, K, T, r)}\left(\tilde{x}_{1}, \ldots, \tilde{x}_{k}\right) \\
& \leqslant g \varepsilon_{1} N^{f(G, H)} \leqslant \frac{g \varepsilon_{0}}{g\left(1+\varepsilon_{0}\right)} c N^{f(G, H)} \\
& \leqslant \frac{\varepsilon_{0}}{1+\varepsilon_{0}} \mathbf{E}_{N, p} N_{(G, H, K, T, r)}\left(\tilde{x}_{1}, \ldots, \tilde{x}_{k}\right) \\
& \leqslant \varepsilon_{0} N_{(G, H, K, T, r)}\left(\tilde{x}_{1}, \ldots, \tilde{x}_{k}\right) .
\end{aligned}
$$

Предложение доказано.

Из этого предложения и неравенств (3), (4) следует, что для любого $r>1$ с асимптотической вероятностью 1 для любых $\tilde{x}_{1}, \ldots, \tilde{x}_{k}$ выполнено неравенство

$$
\begin{aligned}
\mid D_{(G, H, K, T)}\left(\tilde{x}_{1}, \ldots, \tilde{x}_{k}\right)-\mathbf{E}_{N, p} D_{(G, H, K, T)} & \left(\tilde{x}_{1}, \ldots, \tilde{x}_{k}\right) \mid \\
& \leqslant \mathbf{E}_{N, p} d_{(G, H, K, T, 2 r-1)}\left(\tilde{x}_{1}, \ldots, \tilde{x}_{k}\right)+\delta_{r}(N),
\end{aligned}
$$

где

$$
\delta_{r}(N)=o\left(N^{f(G, H)}\right)
$$

Поэтому, в силу предложения 2, для любого $\varepsilon>0$ с асимптотической вероятностью 1 для любых $\tilde{x}_{1}, \ldots, \tilde{x}_{k}$ справедливо неравенство

$$
\left|D_{(G, H, K, T)}\left(\tilde{x}_{1}, \ldots, \tilde{x}_{k}\right)-\mathbf{E}_{N, p} D_{(G, H, K, T)}\left(\tilde{x}_{1}, \ldots, \tilde{x}_{k}\right)\right| \leqslant \varepsilon N^{f(G, H)} .
$$

Такое же неравенство для случайной величины $N_{(G, H)}^{(K, T)}\left(\tilde{x}_{1}, \ldots, \tilde{x}_{k}\right)$ будет напрямую следовать из предложения 5. Чтобы вывести из него утверждение теоремы, нужно доказать, что среднее $\mathbf{E}_{N, p} N_{(G, H)}^{(K, T)}\left(\tilde{x}_{1}, \ldots, \tilde{x}_{k}\right)$ не есть $o\left(N^{f(G, H)}\right)$. 
Предложение 5. Справедливо равенство

$$
\mathbf{E}_{N, p} N_{(G, H)}^{(K, T)}\left(\tilde{x}_{1}, \ldots, \tilde{x}_{k}\right)=\Theta\left(N^{f(G, H)}\right) .
$$

Доказательство. Для доказательства мы воспользуемся теоремой о монотонных свойствах (см. [2]), которая заключается в следующем. Пусть $\mathscr{A}, \mathscr{P} \in \mathscr{F}_{N}-$ возрастающие (убывающие) свойства. Напомним, что множество $\mathscr{A}$ называется возрастающим (соответственно убывающим) свойством, если из того, что $H \in \mathscr{A}$ и $E(G) \supseteq E(H)$ (соответственно $E(G) \subseteq E(H))$ следует, что $G \in \mathcal{A}$. Тогда справедливо неравенство

$$
\mathbf{P}_{N, p}(\mathscr{A} \wedge \mathscr{B}) \geqslant \mathbf{P}_{N, p}(\mathscr{A}) \mathbf{P}_{N, p}(\mathscr{B}) \text {. }
$$

Для каждого множества $\mathscr{B} \in \Omega_{N}$ обозначим $\overline{\mathscr{B}}$ дополнение к множеству $\mathscr{B}$, то есть множество $\Omega_{N} \backslash \mathscr{B}$.

Зафиксируем вершины $\tilde{x}_{1}, \ldots, \tilde{x}_{k} \in \mathscr{V}$. Определим некоторое множество индексов $I\left(\tilde{x}_{1}, \ldots, \tilde{x}_{k}\right)$. Пусть $I\left(\tilde{x}_{1}, \ldots, \tilde{x}_{k}\right)=\{1, \ldots, \mu\}$, где $\mu-$ наибольшее число, при котором выполняется описанное ниже свойство. Каждому индексу $i \in I\left(\tilde{x}_{1}, \ldots, \tilde{x}_{k}\right)$ можно поставить в соответствие упорядоченный набор вершин $\tilde{x}_{k+1}^{i}, \ldots, \tilde{x}_{l}^{i} \in \mathscr{V} \backslash\left\{\tilde{x}_{1}, \ldots, \tilde{x}_{k}\right\}$ (разным индексам соответствуют разные наборы) с единственным следующим ограничением. Пусть $i, j \in I\left(\tilde{x}_{1}, \ldots, \tilde{x}_{k}\right), i \neq j$, наборы $\tilde{x}_{k+1}^{i}, \ldots, \tilde{x}_{l}^{i}$ и $\tilde{x}_{k+1}^{j}, \ldots, \tilde{x}_{l}^{j}$ совпадают (в них входят одни и те же вершины) и для некоторых графов $\mathscr{G}_{1}, \mathscr{G}_{2} \in \Omega_{N}$ графы

$$
\tilde{G}_{1}=\left.\mathscr{G}_{1}\right|_{\left\{\tilde{x}_{1}, \ldots, \tilde{x}_{k}, \tilde{x}_{k+1}^{i}, \ldots, \tilde{x}_{l}^{i}\right\}}, \quad \widetilde{G}_{2}=\left.\mathscr{G}_{2}\right|_{\left\{\tilde{x}_{1}, \ldots, \tilde{x}_{k}, \tilde{x}_{k+1}^{j}, \ldots, \tilde{x}_{l}^{j}\right\}}
$$

являются точными $(G, H)$-расширениями графов

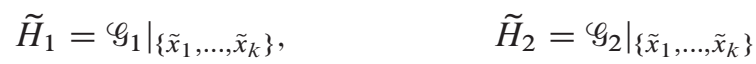

соответственно. Тогда при применении к вершинами $\tilde{x}_{k+1}^{i}, \ldots, \tilde{x}_{l}^{i}$ перенумерации (которая оставляет на месте ребра), переводящей их в $\tilde{x}_{k+1}^{j}, \ldots, \tilde{x}_{l}^{j}$, полученное из $E\left(\widetilde{G}_{1}\right) \backslash E\left(\widetilde{H}_{1}\right)$ множество ребер не совпадает с множеством $E\left(\widetilde{G}_{2}\right) \backslash E\left(\tilde{H}_{2}\right)$. Понятно, что

$$
\mu=\left(\begin{array}{c}
N-k \\
l-k
\end{array}\right) \frac{(l-k) !}{a(G, H)},
$$

где $a(G, H)$ означает число автоморфизмов множества $E(G) \backslash E(H)$. Для каждого $i \in$ $I\left(\tilde{x}_{1}, \ldots, \tilde{x}_{k}\right)$ набор $\tilde{x}_{1}, \ldots, \tilde{x}_{k}, \tilde{x}_{k+1}^{i}, \ldots, \tilde{x}_{l}^{i}$ обозначим $\tilde{\mathbf{x}}^{i}$.

Зафиксируем вершины $\tilde{x}_{1}, \ldots, \tilde{x}_{k}, \tilde{x}_{k+1}, \ldots, \tilde{x}_{l} \in \mathscr{V}$ и определим множество индексов $J\left(\tilde{x}_{1}, \ldots, \tilde{x}_{k}, \tilde{x}_{k+1}, \ldots, \tilde{x}_{l}\right)=\{1, \ldots, v\}$. Пусть $\tilde{x}_{i_{1}}, \ldots, \tilde{x}_{i_{m}} \in\left\{\tilde{x}_{1}, \ldots, \tilde{x}_{k}, \tilde{x}_{k+1}, \ldots, \tilde{x}_{l}\right\}$. Каждому индексу $j \in J\left(\tilde{x}_{1}, \ldots, \tilde{x}_{l}\right)$ можно поставить в соответствие упорядоченный набор вершин $\tilde{x}_{l+1}^{j}, \ldots, \tilde{x}_{l+t}^{j} \in \mathscr{V} \backslash\left\{\tilde{x}_{1}, \ldots, \tilde{x}_{l}\right\}$ с единственным следующим ограничением. Пусть $i, j \in J\left(\tilde{x}_{1}, \ldots, \tilde{x}_{l}\right), i \neq j$, наборы $\tilde{x}_{l+1}^{i}, \ldots, \tilde{x}_{l+t}^{i}$ и $\tilde{x}_{l+1}^{j}, \ldots, \tilde{x}_{l+t}^{j}$ совпадают (в них входят одни и те же вершины), и для некоторых графов $\mathscr{G}_{1}, \mathscr{G}_{2} \in \Omega_{N}$ графы

$$
\widetilde{G}_{1}=\left.\mathscr{G}_{1}\right|_{\left\{\tilde{x}_{i_{1}}, \ldots, \tilde{x}_{i_{m}}, \tilde{x}_{l+1}^{i}, \ldots, \tilde{x}_{t}^{i}\right\}}, \quad \widetilde{G}_{2}=\left.\mathscr{G}_{2}\right|_{\left\{\tilde{x}_{i_{1}}, \ldots, \tilde{x}_{i_{m}}, \tilde{x}_{l+1}^{j}, \ldots, \tilde{x}_{l+t}^{j}\right\}}
$$

являются точными $(K, T)$-расширениями графов

$$
\tilde{T}_{1}=\left.\mathscr{G}_{1}\right|_{\left\{\tilde{x}_{i_{1}}, \ldots, \tilde{x}_{i_{m}}\right\}}, \quad \tilde{T}_{2}=\left.\mathscr{G}_{2}\right|_{\left\{\tilde{x}_{i_{1}}, \ldots, \tilde{x}_{i_{m}}\right\}}
$$


соответственно. Тогда при применении к вершинам $\tilde{x}_{l+1}^{i}, \ldots, \tilde{x}_{l+t}^{i}$ перенумерации (которая оставляет на месте ребра), переводящей их в $\tilde{x}_{l+1}^{j}, \ldots, \tilde{x}_{l+t}^{j}$, полученное из $E\left(\widetilde{G}_{1}\right) \backslash E\left(\tilde{H}_{1}\right)$ множество ребер не совпадает с множеством $E\left(\widetilde{G}_{2}\right) \backslash E\left(\tilde{H}_{2}\right)$. Мощность $J\left(\tilde{x}_{1}, \ldots, \tilde{x}_{l}\right)$, опять же, является максимальной при описанном условии. Понятно, что

$$
v=\left(\begin{array}{c}
N-l \\
t
\end{array}\right) \frac{t !}{a(K, T)} .
$$

Пусть $\mathscr{A}_{i}\left(\tilde{x}_{1}, \ldots, \tilde{x}_{k}\right) \subset \Omega_{N}$, где $i \in I\left(\tilde{x}_{1}, \ldots, \tilde{x}_{k}\right)$, есть множества графов, подграфы которых, индуцированные на множества $\left\{\tilde{x}_{1}, \ldots, \tilde{x}_{k}, \tilde{x}_{k+1}^{i}, \ldots, \tilde{x}_{l}^{i}\right\}$, являются точными $(G, H)$-расширениями подграфов, индуцированных на множество $\left\{\tilde{x}_{1}, \ldots, \tilde{x}_{k}\right\}$; пусть $\mathscr{B}_{j}\left(\tilde{x}_{i_{1}}, \ldots, \tilde{x}_{i_{m}}\right) \subset \Omega_{N}$, где $j \in J\left(\tilde{x}_{1}, \ldots, \tilde{x}_{l}\right)$, есть множества графов, подграфы которых, индуцированные на множества $\left\{\tilde{x}_{i_{1}}, \ldots, \tilde{x}_{i_{m}}, \tilde{x}_{l+1}^{j}, \ldots, \tilde{x}_{l+t}^{j}\right\}$, являются точными $(K, T)$-расширениями подграфов, индуцированных на множество $\left\{\tilde{x}_{i_{1}}, \ldots, \tilde{x}_{i_{m}}\right\}$; и пусть $\mathscr{C}_{j}\left(\tilde{x}_{i_{1}}, \ldots, \tilde{x}_{i_{m}}\right) \subset \Omega_{N}$, где $j \in J\left(\tilde{x}_{1}, \ldots, \tilde{x}_{l}\right)$, есть множества графов, подграфы которых, индуцированные на множества $\left\{\tilde{x}_{i_{1}}, \ldots, \tilde{x}_{i_{m}}, \tilde{x}_{l+1}^{j}, \ldots, \tilde{x}_{l+t}^{j}\right\}$, являются $(K, T)$-расширениями подграфов, индуцированных на множество $\left\{\tilde{x}_{i_{1}}, \ldots, \tilde{x}_{i_{m}}\right\}$.

Для каждого $j \in J\left(\tilde{x}_{1}, \ldots, \tilde{x}_{l}\right)$ введем множество $\widehat{\mathscr{B}}_{j}\left(\tilde{x}_{1}, \ldots, \tilde{x}_{k}, \tilde{x}_{k+1}, \ldots, \tilde{x}_{l}\right) \subset \Omega_{N}$ (множество $\hat{\mathscr{C}}_{j}\left(\tilde{x}_{1}, \ldots, \tilde{x}_{k}, \tilde{x}_{k+1}, \ldots, \tilde{x}_{l}\right) \subset \Omega_{N}$ ), равное объединению по всем упорядоченным наборам вершин $\tilde{x}_{i_{1}}, \ldots, \tilde{x}_{i_{m}} \in\left\{\tilde{x}_{1}, \ldots, \tilde{x}_{k}, \tilde{x}_{k+1}, \ldots, \tilde{x}_{l}\right\}$, удовлетворяющим условию $\left\{\tilde{x}_{i_{1}}, \ldots, \tilde{x}_{i_{m}}\right\} \cap\left\{\tilde{x}_{1}, \ldots, \tilde{x}_{k}\right\} \neq\left\{\tilde{x}_{i_{1}}, \ldots, \tilde{x}_{i_{m}}\right\}$, множеств $\mathscr{B}_{j}\left(\tilde{x}_{i_{1}}, \ldots, \tilde{x}_{i_{m}}\right)$ (множеств $\left.\mathscr{C}_{j}\left(\tilde{x}_{i_{1}}, \ldots, \tilde{x}_{i_{m}}\right)\right)$. Тогда множества $\widehat{\widehat{C}}_{j}\left(\tilde{x}_{1}, \ldots, \tilde{x}_{k}, \tilde{x}_{k+1}, \ldots, \tilde{x}_{l}\right)$ являются убывающими свойствами. Легко заметить, что пересечения монотонных свойств являются мо-

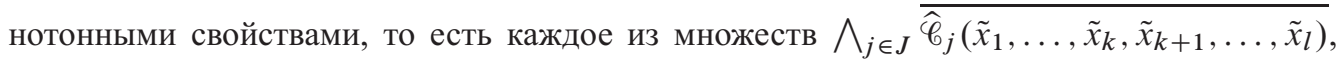
$J \subseteq J\left(\tilde{x}_{1}, \ldots, \tilde{x}_{l}\right)$, является убывающим свойством. Поэтому, в силу теоремы о монотонных свойствах, существуют положительные константы $C_{1}, C_{2}, C_{3}, C_{4}$, для которых при достаточно больших $N$ справедливы соотношения

$$
\begin{aligned}
\mathbf{E}_{N, p} N_{(G, H)}^{(K, T)}\left(\tilde{x}_{1}, \ldots, \tilde{x}_{k}\right) & =\sum_{i \in I\left(\tilde{x}_{1}, \ldots, \tilde{x}_{k}\right)} \mathbf{P}_{N, p}\left(\mathscr{A}_{i}\left(\tilde{x}_{1}, \ldots, \tilde{x}_{k}\right)\right) \mathbf{P}_{N, p}\left(\bigwedge_{j \in J\left(\tilde{\mathbf{x}}^{i}\right)} \overline{\widehat{\mathscr{B}}_{j}\left(\tilde{\mathbf{x}}^{i}\right)}\right) \\
& \sim \sum_{i \in I\left(\tilde{x}_{1}, \ldots, \tilde{x}_{k}\right)} p^{e(G, H)} \mathbf{P}_{N, p}\left(\bigwedge_{j \in J\left(\tilde{\mathbf{x}}^{i}\right)} \overline{\widehat{\mathscr{S}}_{j}\left(\tilde{\mathbf{x}}^{i}\right)}\right) \\
\geqslant & \sum_{i \in I\left(\tilde{x}_{1}, \ldots, \tilde{x}_{k}\right)} p^{e(G, H)} \mathbf{P}_{N, p}\left(\bigwedge_{j \in J\left(\tilde{\mathbf{x}}^{i}\right)} \overline{\hat{\mathscr{C}}_{j}\left(\tilde{\mathbf{x}}^{i}\right)}\right) \\
\geqslant & \sum_{i \in I\left(\tilde{x}_{1}, \ldots, \tilde{x}_{k}\right)} p^{e(G, H)} \prod_{j \in J\left(\tilde{\mathbf{x}}^{i}\right)} \mathbf{P}_{N, p}\left(\overline{\left.\hat{\mathscr{C}}_{j}\left(\tilde{\mathbf{x}}^{i}\right)\right)}\right. \\
\geqslant & C_{1} N^{f(G, H)}\left(1-C_{2} p^{t / \alpha}\right)^{\left(\begin{array}{c}
N \\
t
\end{array}\right)} \\
\geqslant & C_{1} N^{f(G, H)} \exp \left(-C_{3} N^{t} p^{t / \alpha}\right)=C_{4} N^{f(G, H)} .
\end{aligned}
$$

Предложение доказано.

Итак, для обоснования неравенства

$$
\left|N_{(G, H)}^{(K, T)}\left(\tilde{x}_{1}, \ldots, \tilde{x}_{k}\right)-\mathbf{E}_{N, p} N_{(G, H)}^{(K, T)}\left(\tilde{x}_{1}, \ldots, \tilde{x}_{k}\right)\right| \leqslant \varepsilon N^{f(G, H)},
$$


которое, в силу предложения 4, завершит доказательство теоремы, осталось сформулировать и доказать следующий результат.

Предложение 6. Для любого $r \in \mathbf{N}$ с асимптотической вероятностью 1 для любых вериин $\tilde{x}_{1}, \ldots, \tilde{x}_{k}$ справедливо соотночение

$$
N_{(G, H)}\left(\tilde{x}_{1}, \ldots, \tilde{x}_{k}\right)-N_{(G, H)}^{(K, T)}\left(\tilde{x}_{1}, \ldots, \tilde{x}_{k}\right) \sim D_{(G, H, K, T)}\left(\tilde{x}_{1}, \ldots, \tilde{x}_{k}\right) .
$$

Доказательство. Подобное рассуждение мы уже проводили несколько раз - в доказательстве леммы 5, теоремы 3, предложения 4. Заключается оно в том, что графы, образующие нейтральные пары и не обладающие свойством отделимости, формируют жесткую пару.

Случайная величина $N_{(G, H)}\left(\tilde{x}_{1}, \ldots, \tilde{x}_{k}\right)-N_{(G, H)}^{(K, T)}\left(\tilde{x}_{1}, \ldots, \tilde{x}_{k}\right)-D_{(G, H, K, T)}\left(\tilde{x}_{1}, \ldots, \tilde{x}_{k}\right)$ для каждого графа $\mathscr{G} \in \Omega_{N}$ равна сумме двух слагаемых. Первое - это число таких не точных (с дополнительными ребрами) $(G, H)$-расширений $\widetilde{G}$ графа $\widetilde{H}=G_{\left\{\tilde{x}_{1}, \ldots, \tilde{x}_{k}\right\}}$, что пара $(\widetilde{G}, \widetilde{H})$ не является $(K, T)$-максимальной в $\mathscr{G}$. В силу теоремы 4, это второе слагаемое есть $o\left(N^{f(G, H)}\right)$ для подмножества в $\Omega_{N}$, мера $\mathbf{P}_{N, p}$ которого стремится к 1 . Второе слагаемое - это число точных $(G, H)$-расширений $\widetilde{G}$ графа $\tilde{H}$, для которых существуют такие подграфы $\widetilde{K}_{1}, \widetilde{K}_{2}, \widetilde{T}_{1}, \widetilde{T}_{2}$ графа $\mathscr{G}$, что

$$
\begin{array}{rlrl}
\widetilde{T}_{1} & \subset \widetilde{G}, & \widetilde{T}_{2} & \subset \widetilde{G}, \\
\widetilde{T}_{1} \cap \tilde{H} & \neq \widetilde{T}_{1}, & \widetilde{T}_{2} \cap \tilde{H} & \neq \widetilde{T}_{2}, \\
\widetilde{K}_{1} \cap \widetilde{G}=\widetilde{T}_{1}, & & \widetilde{K}_{2} \cap \widetilde{G}=\widetilde{T}_{2},
\end{array}
$$

граф $\widetilde{K}_{1}$ - точное $(K, T)$-расширение графа $\widetilde{T}_{1}$, граф $\widetilde{K}_{2}-$ точное $(K, T)$-расширение графа $\widetilde{T}_{2}$, графы $\widetilde{K}_{1}, \widetilde{K}_{2}$ не обладают свойством отделимости. Пусть $\widetilde{K}_{1}, \widetilde{K}_{2}, \widetilde{T}_{1}, \widetilde{T}_{2}-$ такие графы. Тогда пара $\left(\widetilde{K}_{1} \cup \widetilde{K}_{2}, \widetilde{T}_{1} \cup \widetilde{T}_{2}\right)$ является $\alpha$-жесткой, и по лемме 10.7 .6 в [2] (см. также доказательство предложения 3) с асимптотической вероятностью 1 для любых вершин $\tilde{x}_{1}, \ldots, \tilde{x}_{k}$ справедливо равенство

$$
N_{(G, H)}\left(\tilde{x}_{1}, \ldots, \tilde{x}_{k}\right)-N_{(G, H)}^{(K, T)}\left(\tilde{x}_{1}, \ldots, \tilde{x}_{k}\right)-D_{(G, H, K, T)}\left(\tilde{x}_{1}, \ldots, \tilde{x}_{k}\right)=o\left(N^{f(G, H)}\right) .
$$

Предложение доказано.

\section{4. Доказательство теоремы 2}

Как отмечалось выше, доказательство теоремы 2 мы будем проводить индукцией по числу различных $\alpha$-нейтральных пар $\left(K_{i}, T_{i}\right)$. Мы доказали предложение 1 и тем самым обосновали базу индукции.

Итак, $s \in \mathbf{N}$ и с асимптотической вероятностью 1 для любых вершин $x_{1}, \ldots, x_{k}$

$$
N_{(G, H)}^{\left(K_{1}, T_{1}\right), \ldots,\left(K_{s}, T_{S}\right)}\left(x_{1}, \ldots, x_{k}\right) \sim \mathbf{E}_{N, p} N_{(G, H)}^{\left(K_{1}, T_{1}\right), \ldots,\left(K_{S}, T_{S}\right)}\left(x_{1}, \ldots, x_{k}\right) .
$$

Мы хотим доказать, что с асимптотической вероятностью 1 для любых вершин $x_{1}, \ldots, x_{k}$

$$
N_{(G, H)}^{\left(K_{1}, T_{1}\right), \ldots,\left(K_{s}, T_{s}\right),\left(K_{s+1}, T_{s+1}\right)}\left(x_{1}, \ldots, x_{k}\right) \sim \mathbf{E}_{N, p} N_{(G, H)}^{\left(K_{1}, T_{1}\right), \ldots,\left(K_{S}, T_{s}\right),\left(K_{s+1}, T_{s+1}\right)}\left(x_{1}, \ldots, x_{k}\right) .
$$

Дадим определение $\left\{\left(K_{1}, T_{1}\right), \ldots,\left(K_{s}, T_{s}\right)\right\}$-максимальной $\left(G, H, K_{s+1}, T_{s+1}, r\right)$-сети в графе $\Gamma$.

Пусть графы $H, G, K_{1}, T_{1}, \ldots, K_{s+1}, T_{s+1}$ обладают следующими свойствами: 
- $H \subset G, T_{i} \subset K_{i},\left|V\left(T_{i}\right)\right| \leqslant|V(G)| ;$

- пара $(G, H)$ является $\alpha$-надежной; пары $\left(K_{i}, T_{i}\right)$ являются $\alpha$-нейтральными.

Рассмотрим графы $\widetilde{H}, \widetilde{G}, \widetilde{K}, \widetilde{T}$, а также содержащий их граф $\Gamma$.

Пусть

$$
|V(\widetilde{H})|=|V(H)|=k, \quad \widetilde{T} \subseteq \widetilde{G}, \quad \widetilde{K} \cap \widetilde{G}=\widetilde{T}, \quad \widetilde{H} \cap \widetilde{T} \neq \widetilde{T}
$$

$\widetilde{G}$ - точное $(G, H)$-расширение графа $\widetilde{H}, \widetilde{K}-$ точное $\left(K_{r+1}, T_{r+1}\right)$-расширение графа $\widetilde{T}$;

$$
E((\tilde{K} \cup \widetilde{G}) \backslash \tilde{T}) \backslash(E(\tilde{K} \backslash \tilde{T}) \cup E(\widetilde{G} \backslash \tilde{T}))=\varnothing
$$

пара $(\widetilde{G}, \widetilde{H})$ является $\left(K_{1}, T_{1}\right)$-максимальной, $\ldots,\left(K_{s}, T_{s}\right)$-максимальной в $\Gamma$.

Набор таких графов $(\Gamma, \widetilde{G}, \widetilde{H}, \widetilde{K}, \widetilde{T})$ будем называть $\left\{\left(K_{1}, T_{1}\right), \ldots,\left(K_{s}, T_{s}\right)\right\}$-максимальной $\left(G, H, K_{S+1}, T_{S+1}, 1\right)$-сетью в $\Gamma$.

Если $\left(\Gamma, \widetilde{G}, \widetilde{H}, \widetilde{K}_{1}, \widetilde{T}_{1}\right), \ldots,\left(\Gamma, \widetilde{G}, \widetilde{H}, \widetilde{K}_{r}, \widetilde{T}_{r}\right)$ есть $\left\{\left(K_{1}, T_{1}\right), \ldots,\left(K_{s}, T_{s}\right)\right\}$-максимальные $\left(G, H, K_{s+1}, T_{s+1}, 1\right)$-сети в $\Gamma$, между любыми двумя графами из множества $\left\{\widetilde{K}_{i} \backslash \widetilde{T}_{i}\right\}_{i \in\{1, \ldots, r\}}$ нет ребер, и для всех $i \neq j$ множества $V\left(\widetilde{K}_{i} \backslash \widetilde{T}_{i}\right)$ и $V\left(\widetilde{K}_{j} \backslash \widetilde{T}_{j}\right)$ не пересекаются, то набор $\left(\Gamma, \widetilde{G}, \widetilde{H}, \widetilde{K}_{i}, \widetilde{T}_{i}, i \in\{1, \ldots, r\}\right)$ называют $\left\{\left(K_{1}, T_{1}\right), \ldots,\left(K_{s}, T_{s}\right)\right\}$ максимальной $\left(G, H, K_{s+1}, T_{s+1}, r\right)$-сетью в $\Gamma$.

Дальнейшее доказательство практически дословно повторяет доказательство теоремы 2 , если заменить в рассуждении $(G, H, K, T, r)$-сети на $\left\{\left(K_{1}, T_{1}\right), \ldots,\left(K_{s}, T_{s}\right)\right\}$-максимальные $\left(G, H, K_{s+1}, T_{s+1}, r\right)$-сети в $\Gamma$. Так, в предложении 1 после такой замены, как и в доказательстве, больше ничего менять не придется. В доказательстве предложения 2 вместо теоремы 4 будет применяться индуктивное предположение (причем для точных $(G, H)$-расширений (см. теорему 6 в разделе 4); такая теорема тоже будет верна, это видно из доказательства теоремы 2), вычислительная часть доказательства вообще пропадет, потому что оценка будет вытекать из самого предложения 2 для $(G, H, K, T, r)$ сетей. Предложение 3 не придется менять, так же, как предложение 1. В предложениях 4 и 5 останется заменить случайные величины $N_{(G, H)}^{(K, T)}\left(\tilde{x}_{1}, \ldots, \tilde{x}_{k}\right)$ на величины $N_{(G, H)}^{\left(K_{1}, T_{1}\right), \ldots,\left(K_{S}, T_{s}\right),\left(K_{s+1}, T_{s+1}\right)}\left(\tilde{x}_{1}, \ldots, \tilde{x}_{k}\right)$. Кроме того, в предложении 5 нужно заменить $N_{(G, H)}\left(\tilde{x}_{1}, \ldots, \tilde{x}_{k}\right)$ на $N_{(G, H)}^{\left(K_{1}, T_{1}\right), \ldots,\left(K_{s}, T_{s}\right)}\left(\tilde{x}_{1}, \ldots, \tilde{x}_{k}\right)$.

Теорема доказана.

\section{4. Обобщения}

Для различных пар $\left(K_{1}, T_{1}\right), \ldots,\left(K_{s}, T_{s}\right),(G, H)$, наряду с уже рассмотренными случайными величинами $N_{(G, H)}^{\left(K_{1}, T_{1}\right), \ldots,\left(K_{s}, T_{s}\right)}\left(\tilde{x}_{1}, \ldots, \tilde{x}_{k}\right)$, равными числу некоторых $(G, H)$ расширений, естественно рассмотреть величины $\widehat{N}_{(G, H)}^{\left(K_{1}, T_{1}\right), \ldots,\left(K_{s}, T_{s}\right)}\left(\tilde{x}_{1}, \ldots, \tilde{x}_{k}\right)$, равные числу некоторых точных $(G, H)$-расширений, а именно, пусть, как и раньше, для графов $H$ и $G, H \subset G$, справедливы равенства $V(H)=\left\{x_{1}, \ldots, x_{k}\right\}, V(G)=\left\{x_{1}, \ldots, x_{l}\right\}$. Рассмотрим случайный граф $G(N, p)$ и зафиксируем вершины $\tilde{x}_{1}, \ldots, \tilde{x}_{k} \in \mathscr{V}$. Пусть случайная величина $\hat{R}_{(G, H)}^{\left(K_{1}, T_{1}\right), \ldots,\left(K_{S}, T_{S}\right)}\left(\tilde{x}_{1}, \ldots, \tilde{x}_{k}\right)$ ставит в соответствие каждому графу $\varphi \in \Omega_{N}$ число таких точных $(G, H)$-расширений $\widetilde{G}$ графа $\tilde{H}=\left.\mathscr{G}\right|_{\left\{\tilde{x}_{1}, \ldots, \tilde{x}_{k}\right\}}$, что пара $(\widetilde{G}, \tilde{H})$ является $\left\{\left(K_{1}, T_{1}\right), \ldots,\left(K_{s}, T_{s}\right)\right\}$-максимальной в $\mathscr{G}$. Случайная величина $N_{(G, H)}^{\left(K_{1}, T_{1}\right), \ldots,\left(K_{S}, T_{S}\right)}$ отличается от нее тем, что равна числу всех $(G, H)$-расширений, не только точных. Для 
такой случайной величины справедлив результат, аналогичный теореме 2 (число не точных расширений бесконечно мало по сравнению с числом всех расширений, а число максимальных расширений имеет тот же порядок, что и число всех расширений).

Пусть $\mathcal{N}(r)$ - множество всех $\alpha$-нейтральных пар вида $\left(K_{i}, T_{i}\right)$, где $\left|V\left(T_{i}\right)\right| \leqslant|V(G)|$, $\left|V\left(K_{i}\right) \backslash V\left(T_{i}\right)\right| \leqslant r$. Пусть случайная величина $N_{(G, H)}^{r}\left(\tilde{x}_{1}, \ldots, \tilde{x}_{k}\right)$ (соответственно величина $\left.\widehat{N}_{(G, H)}^{r}\left(\tilde{x}_{1}, \ldots, \tilde{x}_{k}\right)\right)$ ставит в соответствие каждому графу $\mathscr{G} \in \Omega_{N}$ число таких $(G, H)$-расширений $\widetilde{G}$ графа $\tilde{H}=\left.G\right|_{\left\{\tilde{x}_{1}, \ldots, \tilde{x}_{k}\right\}}$ (соответственно таких точных $(G, H)$ расширений $\widetilde{G}$ графа $\left.\widetilde{H}=\left.\mathscr{G}\right|_{\left\{\tilde{x}_{1}, \ldots, \tilde{x}_{k}\right\}}\right)$, что для каждой пары $\left(K_{i}, T_{i}\right) \in \mathcal{N}(r)$ пара $(\widetilde{G}, \widetilde{H})$ является $\left(K_{i}, T_{i}\right)$-максимальной в $\mathscr{G}$. Для этих случайных величин также справедлив результат, аналогичный теореме 2 (число всех $\alpha$-нейтральных пар, состоящих из графов с ограниченным заранее числом вершин, конечно, поэтому этот результат напрямую следует из теоремы 3 и ее аналога для точных $(G, H)$-расширений).

Теорема 5. Пусть $0<\alpha \leqslant 1$; пара $(G, H)$ является $\alpha$-надежной, и пары $(K, T),\left(K_{1}, T_{1}\right)$, $\ldots,\left(K_{s}, T_{s}\right)$ являются $\alpha$-нейтральными. Пусть, кроме того, $p=N^{-\alpha}$. Тогда с асимптотической вероятностью 1 для любых вершин $\tilde{x}_{1}, \ldots, \tilde{x}_{k}$

$$
\begin{aligned}
\hat{N}_{(G, H)}^{(K, T)}\left(\tilde{x}_{1}, \ldots, \tilde{x}_{k}\right) & \sim \mathbf{E}_{N, p} N_{(G, H)}^{(K, T)}\left(\tilde{x}_{1}, \ldots, \tilde{x}_{k}\right) \\
& =\Theta\left(N^{f(G, H)}\right), \\
\widehat{N}_{(G, H)}^{\left(K_{1}, T_{1}\right),\left(K_{2}, T_{2}\right), \ldots,\left(K_{S}, T_{S}\right)}\left(\tilde{x}_{1}, \ldots, \tilde{x}_{k}\right) & \sim \mathbf{E}_{N, p} N_{(G, H)}^{\left(K_{1}, T_{1}\right),\left(K_{2}, T_{2}\right), \ldots,\left(K_{s}, T_{s}\right)}\left(\tilde{x}_{1}, \ldots, \tilde{x}_{k}\right) \\
& =\Theta\left(N^{f(G, H)}\right), \\
N_{(G, H)}^{r}\left(\tilde{x}_{1}, \ldots, \tilde{x}_{k}\right) & \sim \mathbf{E}_{N, p} N_{(G, H)}^{r}\left(\tilde{x}_{1}, \ldots, \tilde{x}_{k}\right) \\
& =\Theta\left(N^{f(G, H)}\right), \\
\hat{N}_{(G, H)}^{r}\left(\tilde{x}_{1}, \ldots, \tilde{x}_{k}\right) & \sim \mathbf{E}_{N, p} N_{(G, H)}^{r}\left(\tilde{x}_{1}, \ldots, \tilde{x}_{k}\right) \\
& =\Theta\left(N^{f(G, H)}\right) .
\end{aligned}
$$

Сделаем заключительное замечание, объясняющее, что нашими результатами исчерпываются все виды максимальных $\alpha$-надежных пар. Действительно, в соответствии с леммой 2 , для любой пары $(K, T)$ существуют такие графы $X, Y$, что $T \subseteq Y \subseteq X \subseteq K$ и пары $(Y, T),(X, Y)$ и $(K, X)$ являются $\alpha$-жесткой, $\alpha$-нейтральной цепью и $\alpha$-надежной соответственно. Поэтому, если $T \neq Y$, то внутренняя пара является $\alpha$-жесткой и число таких $(K, T)$-расширений $\widetilde{G}$ подграфа в случайном графе, индуцированного на множество вершин $\left\{\tilde{x}_{1}, \ldots, \tilde{x}_{k}\right\}$, что пара $\left(\widetilde{G},\left.\widetilde{G}\right|_{\left\{\tilde{x}_{1}, \ldots, \tilde{x}_{k}\right\}}\right)$ является $(K, T)$-максимальной, больше чем число таких $(G, H)$-расширений $\widetilde{G}$, что пара $\left(\widetilde{G},\left.\widetilde{G}\right|_{\left\{\tilde{x}_{1}, \ldots, \tilde{x}_{k}\right\}}\right)$ является $(Y, T)$ максимальной. Поэтому, в соответствии с леммой 10.7 .6 в [2], число $(K, T)$-максимальных пар с асимптотической вероятностью 1 эквивалентно числу всех $(G, H)$-расширений. Если же $Y=T$, то внутренняя пара является $\alpha$-нейтральной цепью, поэтому существует такой граф $S$, что $T \subset S \subseteq X$ и пара $(S \backslash T \cup Z, Z)$ является $\alpha$-нейтральной для некоторого графа $Z \subseteq T$, причем ни одна вершина графа $T \backslash Z$ не соединена ребром ни с одной вершиной графа $S \backslash T$. Следовательно, число таких $(K, T)$-расширений $\widetilde{G}$ подграфа в случайном графе, индуцированного на множество вершин $\left\{\tilde{x}_{1}, \ldots, \tilde{x}_{k}\right\}$, что пара $\left(\widetilde{G},\left.\widetilde{G}\right|_{\left\{\tilde{x}_{1}, \ldots, \tilde{x}_{k}\right\}}\right)$ является $(K, T)$-максимальной, больше чем число таких $(G, H)$-расширений $\widetilde{G}$, что паpa $\left(\widetilde{G},\left.\widetilde{G}\right|_{\left\{\tilde{x}_{1}, \ldots, \tilde{x}_{k}\right\}}\right)$ является $(S, T)$-максимальной. Поэтому, в соответствии с теоремой 2 , число $(K, T)$-максимальных пар с асимптотической вероятностью 1 равно $\Theta\left(N^{f(G, H)}\right)$. Эти же рассуждения можно провести и для точных $(G, H)$-расширений. 


\section{Список литературы}

1. Spencer J. H., Counting extensions. J. Combinatorial Theory (1990) A55, 247-255.

2. Alon N., Spencer J., Probabilistic method. Wiley, New York, 1992.

3. Shelah S., Spencer J. H., Zero-one laws for sparse random graphs. J. American Math. Soc. (1988) 1, 97-115.

4. McArthur M., The asymptotic behavior of $L_{\infty, \omega}^{k}$ on sparse random graphs. DIMACS (1997) 33, 53-63.

5. Janson S., Luczak T., Rucinski A., Random Graphs. Wiley, New York, 2000.

6. Жуковский М. Е., Ослабленный закон нуля или единицы для случайных дистанционных графов. Доклады РАН (2010) 430, №3, 314-317.

7. Успенский В. А., Верещагин Н. К., Плиско В. Е., Вводный курс математической логики. Физматлит, Москва, 1997.

8. Верещагин Н. К., Шень А., Языки и исчисления. МЦНМО, Москва, 2000.

9. Diestel R., Graph theory. Springer, Berlin, 2000.

10. Harary F., Graph theory. Addison-Wesley, Reading, Mass., 1969.

11. Erdős P., Rényi A., On the evolution of random graphs. Publ. Math. Inst. Hung. Acad. Sci. (1960) 5, 17-61.

12. Bollobás B., Random graphs. Cambridge Univ. Press, Cambridge, 2001.

13. Колчин В. Ф., Случайные графы. Физматлит, Москва, 2004.

Статья поступила 13.10.2010. 